Please do not remove this page

RMIT

UNIVERSITY

\title{
Probabilistic tree-based representation for solving minimum cost integer flow problems with nonlinear non-convex cost functions
}

Ghasemishabankareh, Behrooz; Li, Xiaodong; Ozlen, Melih; Neumann, Frank

https://researchrepository.rmit.edu.au/esploro/outputs/9921861987101341/filesAndLinks?institution=61RMIT_INST\&index=null

Ghasemishabankareh, B., Li, X., Ozlen, M., \& Neumann, F. (2020). Probabilistic tree-based representation for solving minimum cost integer flow problems with nonlinear non-convex cost functions. Applied Soft Computing Journal, 86, 1-14. https://doi.org/10.1016/j.asoc.2019.105951

Document Version: Accepted Manuscript

Published Version: https://doi.org/10.1016/j.asoc.2019.105951

Repository homepage: https://researchrepository.rmit.edu.au

(c) 2019 Elsevier B.V. All rights reserved.

Downloaded On 2023/04/27 01:34:17 +1000 
Thank you for downloading this document from the RMIT Research Repository.

The RMIT Research Repository is an open access database showcasing the research outputs of RMIT University researchers.

RMIT Research Repository: http://researchbank.rmit.edu.au/

\section{Citation:}

Ghasemishabankareh, B, Li, X, Ozlen, M and Neumann, F 2020, 'Probabilistic tree-based representation for solving minimum cost integer flow problems with nonlinear non-convex cost functions', Applied Soft Computing Journal, vol. 86, 105951, pp. 1-14.

See this record in the RMIT Research Repository at:

https://researchbank.rmit.edu.au/view/rmit:56426

Version: Accepted Manuscript

\section{Copyright Statement:}

(C) This work is licensed under a Creative Commons Attribution-NonCommercial-NoDerivs 2.0 Generic License. https://creativecommons.org/licenses/by-nc-nd/2.0/

\section{Link to Published Version:}

https://dx.doi.org/10.1016/j.asoc.2019.105951 


\title{
Probabilistic Tree-Based Representation for Solving Minimum Cost Integer Flow Problems with Nonlinear Non-convex Cost Functions
}

\author{
Behrooz Ghasemishabankareh ${ }^{\mathrm{a}, *}$, Xiaodong $\mathrm{Li}^{\mathrm{a}}$, Melih Ozlen ${ }^{\mathrm{a}}$, Frank \\ Neumann ${ }^{\mathrm{b}}$ \\ ${ }^{a}$ School of Science, RMIT University, Melbourne, Australia \\ ${ }^{b}$ School of Computer Science, The University of Adelaide, Adelaide, Australia
}

\begin{abstract}
The minimum cost flow problem (MCFP) is the most generic variation of the network flow problem which aims to transfer a commodity throughout the network to satisfy demands. The problem size (in terms of the number of nodes and arcs) and the shape of the cost function are the most critical factors when considering MCFPs. Existing mathematical programming techniques often assume the cost functions to be linear or convex. Unfortunately, the linearity and convexity assumptions are too restrictive for modelling many real-world scenarios. In addition, many real-world MCFPs are large-scale, with networks having a large number of nodes and arcs. In this paper, we propose a probabilistic tree-based genetic algorithm (PTbGA) for solving large-scale minimum cost integer flow problems with nonlinear non-convex cost functions. We first compare this probabilistic tree-based representation scheme with the priority-based representation scheme, which is the most commonly-used representation for solving MCFPs. We then compare the performance of PTbGA with that of the priority-based genetic algorithm (PrGA), and two state-of-the-art mathematical solvers on a set of MCFP instances. Our experimental results demonstrate the superiority and efficiency of PTbGA in dealing with large-sized MCFPs, as compared to the PrGA
\end{abstract}

\footnotetext{
*Corresponding author

Email addresses: behrooz.ghasemishabankareh@rmit.edu.au (Behrooz

Ghasemishabankareh ), xiaodong.li@rmit.edu.au (Xiaodong Li),

melih.ozlen@rmit.edu.au (Melih Ozlen), frank.neumann@adelaide.edu.au (Frank Neumann)
} 
method and the mathematical solvers.

Keywords:

Minimum cost flow problem, genetic algorithm, representation scheme, mixed integer nonlinear programming, Taguchi experimental design

\section{Introduction}

Network flow problems have many real-world applications such as electrical and power networks, telecommunications, road and rail networks, airline services networks [1]. The shortest path problem, the maximum flow problem, the assignment problem, the transportation problem, and the minimum cost flow problem (MCFP) are different variations of the network flow problem. Among these, MCFP is the most generic network flow problem with numerous applications such as distribution problems, scheduling, optimal loading of a hopping aeroplane, and racial balancing of schools [1].

The shape of cost function (linear, nonlinear, convex, concave and nonconvex) on each arc, is one of the main factors contributing to the complexity of network flow problems and more specifically MCFPs [2]. The linear MCFP is polynomially solvable [3] by using a strong polynomial algorithm [4]. Two recent surveys for solving MCFPs using linear cost functions are presented in $[5,6]$.

However, in many real-world problems, linearity assumption as well as linear approximation of the nonlinear functions cannot capture the real-world situations adequately. In such cases, ideally the nonlinear cost functions should be directly employed in order to accurately model the real-world scenarios [7]. The non-linearity of cost function occurs due to economies of scale, and it happens when the cost per unit of flow decreases by increasing flow or when there is a fixed charge for sending a flow through a new arc in the network [2]. Many studies in the literature suggest the appropriateness of employing nonlinear cost functions in the network flow problems [8,9] and the power flow and power systems problems $[10,11,12]$.

MCFP considering a concave cost function and its special case called single-source uncapacitated (SSU) MCFP with fixed-charge costs are known to be NP-hard [13]. The complexity of the concave MCFP arises because during minimisation of a concave function over a convex feasible region (defined by the network constraints) a local optimum is not necessarily a global optimum [3]. There are several studies in the literature which solved SSU 
MCFP with a concave cost function in order to find a global optimum or an approximate optimum which serves as a bound on the global optimum. A dynamic programming approach, branch-and-bound method, and a linear approximation of a concave cost function were introduced to solve SSU MCFP using concave cost functions $[14,15,16]$. However, the above-mentioned algorithms are not capable of handling large-scale networks, neither efficiently. For instance, Fontes et al. [14] solved network instances only up to 19 nodes in 5 hours, and Burkard et al. [16] considered networks up to 21 nodes, but it took 13 hours.

Metaheuristic methods are another popular approach for solving the nonlinear network flow problems and more specifically MCFPs. Among these, ant colony optimisation (ACO) and genetic algorithm (GA) are most commonlyused. For example, an ACO algorithm and a hybrid ACO with local search were proposed in $[3,17]$ for tackling the SSU MCFPs. Both methods were able to solve the uncapacitated network instances, with the largest network instances considered being networks with just 50 nodes.

Representation plays an important role in the success of an GA. In order to deal with network flow problems, several representation schemes have been proposed such as variable-length encoding [18], fixed-length encoding [19], priority-based representation (PbR) [20], improved priority-based representation [21], and random key representation [2]. Additionally, several studies have been conducted to solve the unit commitment problems in power systems using priority list representation [10, 22, 23]. Among these, the variablelength [24] and the fixed-length [25] encoding schemes are not equipped for solving MCFP since they produce infeasible solutions.

Random key representation and $\mathrm{PbR}$ are frequently used for combinatorial optimisation. For instance, two genetic algorithm methods using prioritybased representation were proposed to solve transportation problems in [26] and [20]. A random key representation was applied for solving resource constrained project scheduling problem [27] as well as a multi-mode resourceconstrained project scheduling problem [28]. In order to deal with MCFPs, Gen et al. [29] shows that PbR can be applied for solving small-sized bicriteria MCFPs (i.e. network with 25 nodes and 56 arcs). Whereas Fontes et al. [2] show that the random key representation can be effectively used in a hybrid GA for solving MCFPs with concave cost functions. In this case, uncapacitated networks with medium-sized instances (up to 50 nodes) were considered.

The aforementioned studies $[2,27,26,20,30]$ have focused on the unca- 
pacitated MCFPs, however, it would be desirable to solve the capacitated MCFPs directly. Although the capacitated networks can be transformed into uncapacitated ones, the transformation procedure introduces a large number of new arcs and nodes. As a result, it significantly increases the number of decision variables especially when dealing with large-scale problems [1]. Very few attempts have been made to directly handle the capacitated MCFP, with an exception in [31], where a deterministic annealing algorithm was developed for solving the capacitated MCFPs with a dense network (up to 130 variables).

In this paper we propose a probabilistic tree-based genetic algorithm (PTbGA) for solving the large-scale MCFP using nonlinear non-convex cost functions, to tackle the following issues in MCFPs: 1) networks with a large number of arcs and integer flows; 2) various shapes of the cost function; 3) dealing with a capacitated network directly. The proposed PTbGA method allows us to consider the capacitated MCFPs directly without the need of transforming a capacitated network into an uncapacitated one. As a result, PTbGA's capability to solve the large-scale problem instances is much enhanced.

Building on our preliminary work [32], where only single-source and singlesink network instances were considered, this paper provides a more detailed account of the probabilistic tree-based representation (PTbR) scheme, which provides the key ingredients allowing us to search the entire feasible search space more effectively, as compared with the conventional priority-based representation $(\mathrm{PbR})$ scheme. Furthermore, we introduce an improved decoding procedure that allows more thorough sampling of the search space. Finally, we conduct systematic and extensive experiments to compare the performance of the PTbR-based GA (PTbGA) variant with the PbR-based GA (PrGA) for solving multi-source and multi-sink MCFP instances (up to 48,000 arcs), using nonlinear non-convex cost functions. We use the Taguchi method to identify the optimal parameter settings for both PTbGA and PrGA. The performances of these two GA variants are compared with those of the-state-of-the-art mathematical solvers on a set of small, medium, and large-sized network instances. Our experimental results demonstrate the superiority of the PTbGA over the PrGA and the mathematical programming solvers, especially when handling the large-scale MCFPs, as well as its robustness when using a variety of nonlinear non-convex cost functions.

The remainder of the paper is organised as follows: the definition of the MCFP is provided in Section 2. Section 3 presents the proposed PTbR scheme, and compares it with the commonly-used PbR scheme. The pro- 
posed PTbGA method is presented in Section 4. Section 5 describes the problem instances used, parameter settings, and experimental results. Section 6 provides the conclusion.

\title{
Nomenclature and abbreviation
}

\author{
Mathematical symbols \\ $A \quad$ A set of arcs. \\ $b(i) \quad$ Amount of supply and demand for node $i$. \\ $c_{i j} \quad$ Non-negative coefficient in cost function. \\ $\begin{array}{ll}f_{i j} & \text { Cost function on arc }(i, j) \text {. } \\ G & \text { Directed network. }\end{array}$ \\ $l_{i j} \quad$ Lower bound of arc $(i, j)$. \\ $m \quad$ Number of arcs. \\ $n$ Number of nodes. \\ $\mathcal{N} \quad$ A set of nodes. \\ $x_{i j} \quad$ An integer flow from node $i$ to node $j$. \\ $u_{i j} \quad$ Capacity of $\operatorname{arc}(i, j)$.
}

\section{Notations}

Flow $\quad$ Amount of flow can be sent on Path . $_{\text {. }}$

$h \quad$ Indicator for the outcome of one-sample $t$-test.

No. $\quad$ Instance number.

$O B J \quad$ Cost function value obtained by mathematical solvers.

$p \quad$ Path counter.

std Standard deviation of cost function over 30 runs.

$t \quad$ Average running time in seconds.

$t^{\prime} \quad$ Running time for mathematical solvers.

$U \quad$ Capacity of each Path.

\section{Parameters}

$\alpha \quad$ Percentage of population members which are initialised randomly.

$I t_{\max } \quad$ Maximum number of iterations.

$N$ Number of decoding procedure for PTbGA.

$P_{c} \quad$ Crossover rate.

pop_size Number of individuals in each population for GA-based methods.

\section{Abbreviations}

MCFP Minimum cost flow problem.

MINLP Mixed integer nonlinear programming.

NFEs Maximum number of function evaluations.

NLP Nonlinear programming.

OR Operations research.

$\mathrm{PbR} \quad$ Priority-based representation scheme.

PrGA Priority-based genetic algorithm.

PTbGA Probabilistic tree-based genetic.

PTbR Probabilistic tree-based representation scheme.

RPD Relative percentage deviation.

$\mathrm{S} / \mathrm{N} \quad$ Signal-to-noise.

WMX Weight mapping crossover. 


\section{Problem definition}

Let $G=(\mathcal{N}, A)$ be a directed network with a set $\mathcal{N}$ which consists of $n$ nodes and a set $A$ of $m$ arcs. Each arc $(i, j)$ has a capacity of $u_{i j}$ and lower bound of $l_{i j}$ which denote the maximum and minimum amount that can be sent on the $\operatorname{arc}(i, j)$, respectively. Each node $i \in \mathcal{N}$ is associated with an integer value $b(i)$. If $b(i)$ is positive, it shows that node $i$ is a supply node, if $b(i)$ is negative, node $i$ is a demand node with demand of $|b(i)|$ and finally $b(i)=0$ shows the transshipment node $i$. A decision variable in an MCFP is an integer flow and denoted by $x_{i j}$. The associated cost for each flow $\left(x_{i j}\right)$ is denoted by $f_{i j}\left(x_{i j}\right)$. Fig. 1 shows an example of the MCFP with $n=5$ nodes and $m=7$ arcs, which has a supplier node $(b(1)=10)$ and a demand node $(b(5)=-10)$. Generally in MCFP, we aim to send flows throughout the network to satisfy all demands by minimising the total cost (i.e., the objective function value). The formulation of the MCFP is as follows [1]:

$$
\begin{gathered}
\text { Minimise }: z(\overrightarrow{\mathbf{x}})=\sum_{(i, j) \in A} f_{i j}\left(x_{i j}\right), \\
\text { s.t. } \sum_{\{j:(i, j) \in A\}} x_{i j}-\sum_{\{j:(j, i) \in A\}} x_{j i}=b(i) \quad \forall i \in \mathcal{N}, \\
l_{i j} \leq x_{i j} \leq u_{i j} \quad \forall \quad(i, j) \in A, \\
x_{i j} \in \mathbb{Z} \quad \forall \quad(i, j) \in A,
\end{gathered}
$$

where Eq.1 minimises the total cost within the network. Eq. 2 is a flow balance constraint and states that the difference between the total outflow (first term) and the total inflow (second term) is equal to $b(i)$. Eq. 3 ensures that the flow on each arc is between upper and lower bound, and finally Eq. 4 ensures that all flow values are integer. In this study we consider the following assumptions for the MCFP: 1) the lower bounds on all arcs are set to zero; 2) the network is directed; 3) there are no two or more arcs with the same tail or head in the network; 4) the total demand and supply in the network are equal, i.e. $\left.\sum_{i=1}^{n} b(i)=0 ; 5\right)$ there are more than one supplier and one demand node in the network; and 6) the cost function on each arc $\left(f_{i j}\right)$ is a nonlinear non-convex function. 


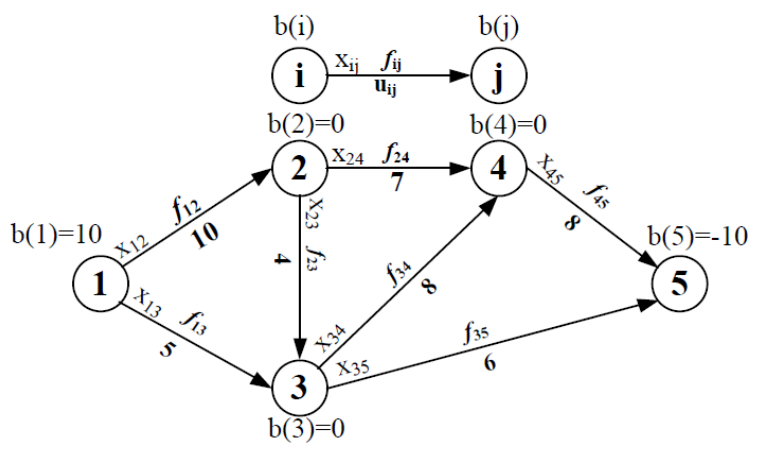

Figure 1: An example of the MCFP $(n=5, m=7)$.

\section{Representation schemes}

A good representation scheme can often make an optimisation problem easier to solve. In the following, we first describe the priority-based representation $(\mathrm{PbR})$ which is the most commonly-used representation method for handling MCFPs and discuss its drawbacks. Then we present the probabilistic tree based representation (PTbR) scheme for solving the MCFP and we demonstrate that this representation scheme can help the search in the right direction in feasible regions of the search space of the MCFP.

\subsection{Priority-based representation (PbR)}

A chromosome in the GA has two main characteristics: locus, the position of a gene in a chromosome; and allele, the possible values each gene can take. To represent a candidate solution for an MCFP, PbR lets the number of genes to be equal to $n$ and the allele is generated randomly between 1 and $n$, which represents the priority of each node for constructing a path among all possible nodes [29]. Fig. 2 illustrates the PbR chromosome and its corresponding solution for the network presented in Fig. 1.

To obtain a feasible solution for a given $\mathrm{PbR}$ chromosome presented, a two-phase decoding procedure is followed. In phase I, a path is generated based on the priorities and the maximum possible flow is sent though the generated path in phase II. The decoding procedure starts from node 1 . Based on the network presented in the Fig. 1, the possible successor nodes are nodes 2 and 3. Based on Fig. 2a, the priorities of nodes 2 and 3 are equal to 5 and 2, respectively. Since node 2 has a higher priority, node 2 is selected as the successor. Node 2's possible successors are nodes 3 and 4 . 


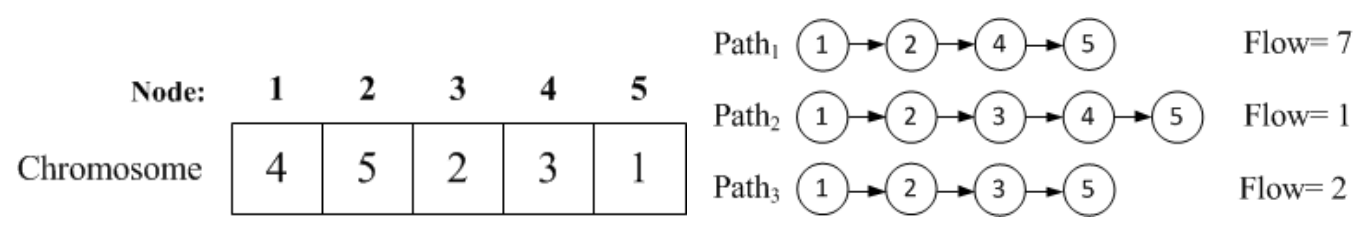

(a) An example of a chromosome for the net- (b) A feasible solution for the given chrowork in Fig. 1. mosome.

Figure 2: The PbR chromosome and its corresponding solution.

Since the priority of node 4(3) is higher than node 3(2), node 4 is selected. Finally from node 4 we can only move to node 5 . Hence, the following path is generated: $1 \rightarrow 2 \rightarrow 4 \rightarrow 5$. In phase II, in order to send a feasible flow on the generated path, the capacity of the generated path is calculated: $U=\min \left\{u_{12}=10, u_{24}=7, u_{45}=8\right\}=7$. Consequently, the flow on the generated path is equal to 7 . After sending the flow on the network, the upper bound $\left(u_{i j}\right)$, supply and demand should be updated. Since the supply/demand is not equal to 0 , the second path should be generated. The above procedure repeats until all demands are satisfied. Fig. 2b presents the feasible solution for the given chromosome in Fig. 2a after executing the decoding procedure.

Although PbR is commonly used in the network flow problems [29], but it has some limitations in representing the full extent of the feasible search space [32]. Fig. 3 shows an example for the network presented in Fig. 1 that $\mathrm{PbR}$ is unable to represent. Here the first path is generated as follows: $1 \rightarrow 2 \rightarrow 4 \rightarrow 5$. Since in $\mathrm{Path}_{1}$ after node 1 , node 2 is selected, it shows that node 2 has a higher priority than node 3 . Hence, if arc $(1,2)$ is not saturated, $\mathrm{PbR}$ will not allow any flow to be sent through arc $(1,3)$, essentially blocking this possibility completely (see $\mathrm{Path}_{2}$ in Fig. 3). This means that $\mathrm{PbR}$ is unable to represent a potential feasible solution such that the flow would go through arc $(1,3)$ (as shown in Fig. 3). Another drawback for $\mathrm{PbR}$ is that each time a path is generated, we are supposed to send the maximum possible amount on the generated path. For instance, for the network in Fig. 1, if the first generated path is Path $1: 1 \rightarrow 2 \rightarrow 4 \rightarrow 5$, since $U=\min \left\{u_{12}=10, u_{24}=7, u_{45}=8\right\}=7$, we are not allowed to send a flow less than 7 . These limitations would restrict a search algorithm from reaching the full extent of the feasible search space. 


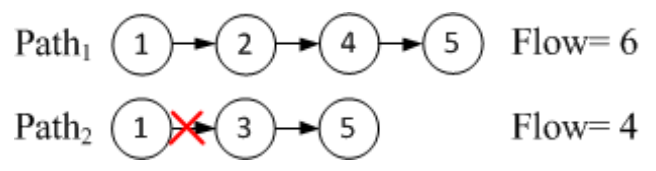

Figure 3: An example of the feasible solution that $\mathrm{PbR}$ fails to represent (for the network in Fig. 1).

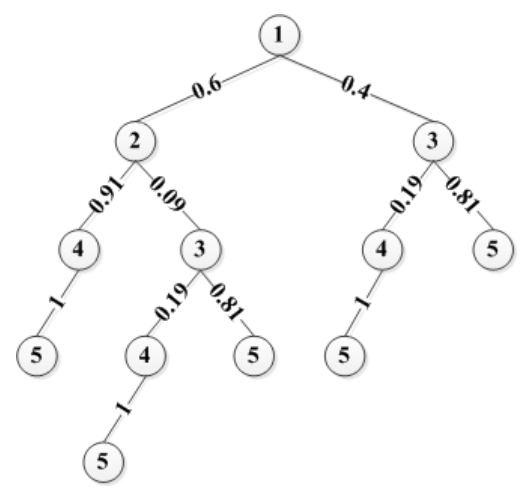

Figure 4: A tree structure for the network presented in Fig. 1

\subsection{Probabilistic tree-based representation (PTbR)}

To counteract the above limitations of the $\mathrm{PbR}$, here we propose a probabilistic tree-based representation (PTbR) scheme, where a probability tree is adopted to represent a potential MCFP solution. Unlike the PbR scheme which is restricted to a small part of the feasible space, the PTbR is able to represent all possible feasible solutions. The PTbR can be represented as a tree structure where the probability of moving from one node to another is defined on the branches of the tree. Fig. 4 illustrates the probability tree structure for the network in Fig. 1.

The tree structure can be converted to a chromosome. Fig. 5 shows an example of a chromosome for the tree structure presented in Fig. 4. The chromosome has $n$ - 1 sub-chromosomes ( $\mathrm{SCh}), m$ genes, and within each sub-chromosome the value of each gene (which represents the probability) is between 0 and 1 and cumulatively increases to one.

Based on Algorithm 1, in order to obtain a feasible solution from the PTbR (e.g. a chromosome), we follow two phases. In the first phase, a path is constructed and in the second phase, a feasible flow is sent through the generated path. This process is repeated until the demand is satisfied. The inputs for Algorithm 1 are chromosome, supply/demand nodes. To obtain a 


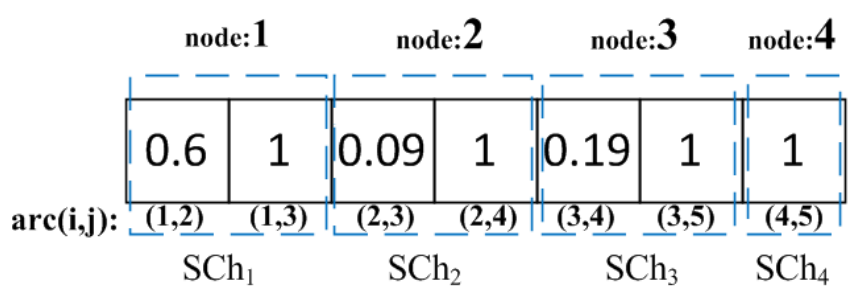

Figure 5: A chromosome for the tree structure in Fig. 4

feasible solution for the chromosome in Fig. 5, we start generating the first path from node $i=1\left(\mathrm{SCh}_{i=1}\right)$. First, a random number is generated in $[0,1]$ (e.g. rand $=0.2$, line 10). Based on the chromosome in Fig. 5, from node 1 (i.e. $\left.\mathrm{SCh}_{i=1}\right)$ we have two outgoing arcs $(1,2)$ and $(1,3)$ which connect us to nodes 2 and 3 respectively. Since the generated random number is between 0 and 0.6 (i.e $0 \leq$ rand $=0.2 \leq 0.6$ ), we reach node 2 by moving through arc $(1,2)$ and node 2 will be added to the generated path (line 11). Since we have not reached to the node $n=5$ (line 9$)$ yet, from node $2\left(\mathrm{SCh}_{i=2}\right)$ another random number is generated $(0.09 \leq$ rand $=0.85 \leq 1)$ and we move through arc $(2,4)$ to reach to node 4 . From node 4 , the only available node is 5 (i.e. through arc $(4,5))$. Since we have already reached node $n=5$, the first phase is terminated and here is the generated path: $1 \rightarrow 2 \rightarrow 4 \rightarrow 5$.

In the Phase II of Algorithm 1, we attempt to send a feasible flow through the generated path. First the capacity of the generated path is defined (line $\left.15, U=\min \left\{u_{12}=10, u_{24}=7, u_{45}=8\right\}=7\right)$. By generating a random integer number in $[1, \min \{U=7, b(1)=|b(n)|=10\}]$ (line 16) a feasible flow for the constructed path is defined (e.g. Flow $w_{p=1}=6$ ). Since the demand has not been fully met (i.e., not equal to 0 yet) (line 3 ), the above procedure (Phase I and II of Algorithm 1) is repeated. The outputs for Algorithm 1 are the generated paths as well as their feasible flows. For instances, Fig. 6 shows a feasible solution with a second path generated for the chromosome presented in Fig. 5. This solution would be impossible to generate from the PbR method (as shown in Fig. 3).

\section{Probabilistic tree-based GA (PTbGA)}

After showing the advantages of adopting PTbR over PbR in generating more freely feasible solutions for the MCFP, this section presents PTbGA (the proposed PTbR-based genetic algorithm), which incorporates PTbR 


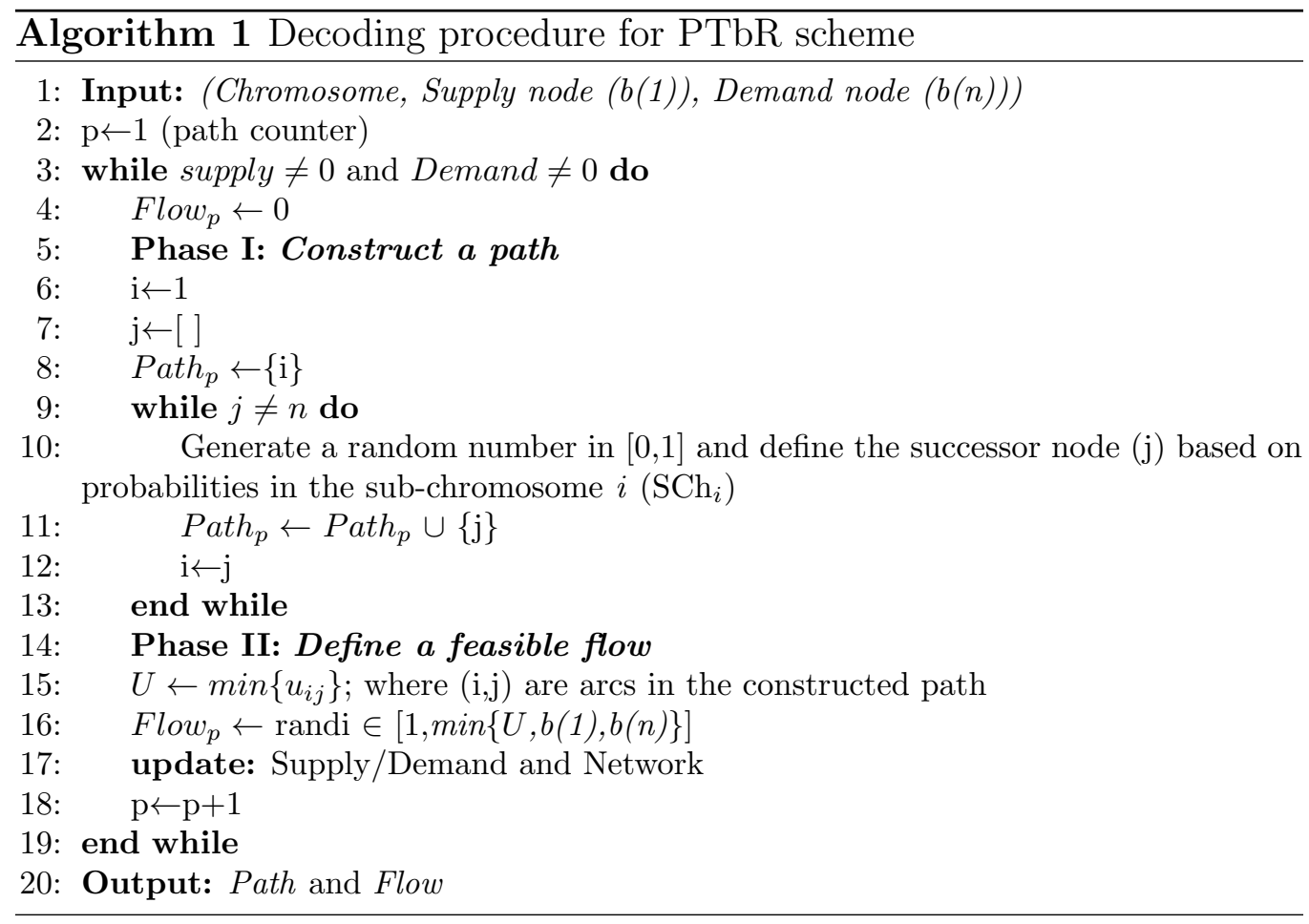

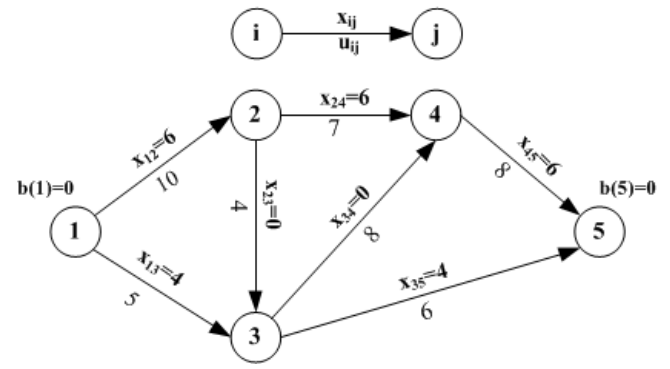

(a) Decision variables in the network.

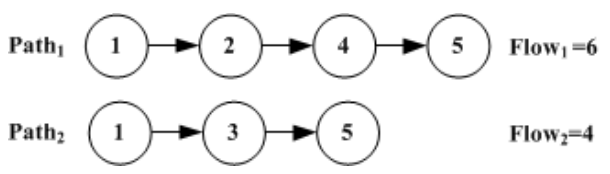

(b) Feasible paths and flows.

Figure 6: An example of a feasible solution for the sample chromosome in Fig. 5.

into an GA. Our aim is to evolve a population of probabilistic trees, so that it will not only allow us to freely produce more feasible MCFP solutions, but also ultimately better solutions at the end of an optimisation run. Algorithm 2 summarises the procedure of PTbGA. 

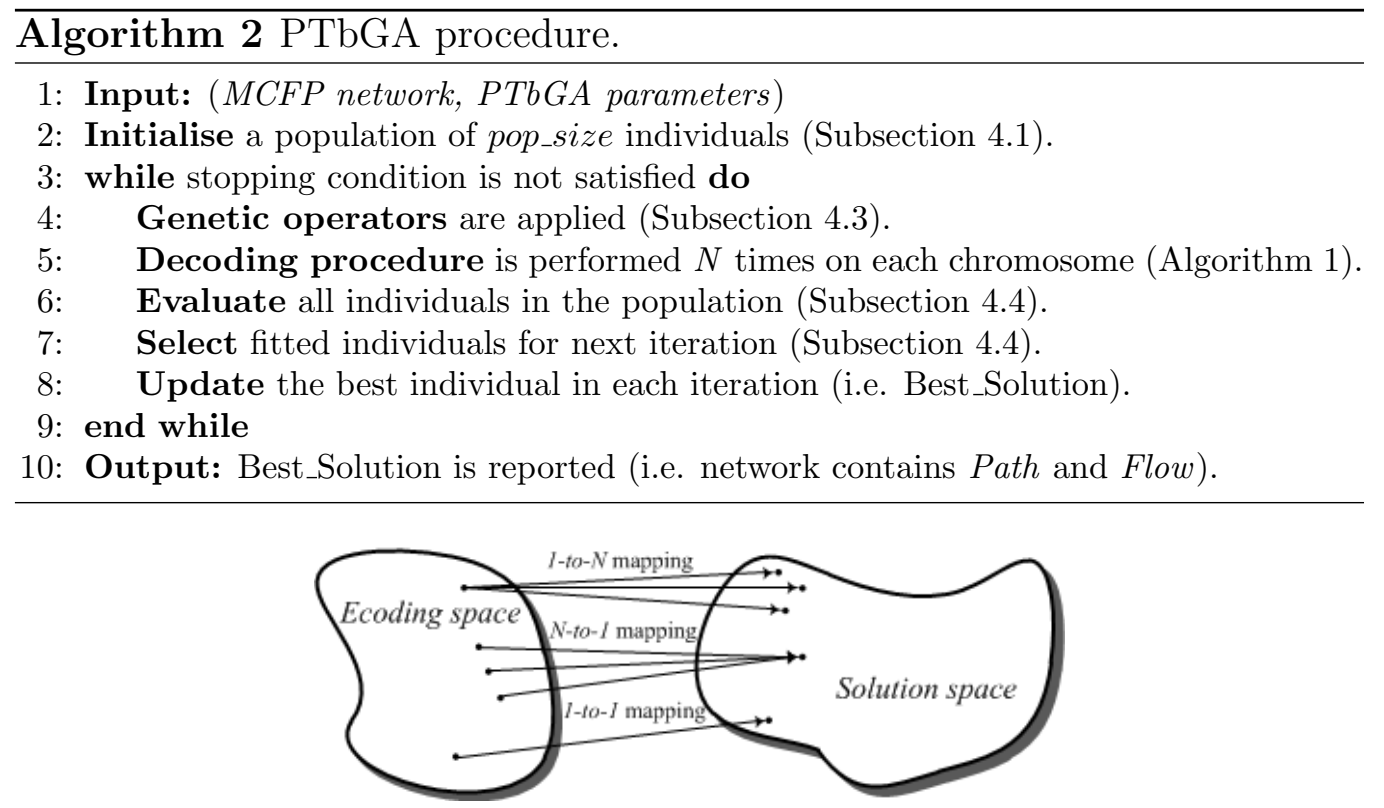

Figure 7: The mapping from the encoding space to the solution space.

\subsection{Initialisation}

At the start of PTbGA, a population with pop_size individuals is generated. Instead of applying the $100 \%$ random initialisation, $\alpha \%$ of the population members are generated randomly and (1- $\alpha) \%$ members of pop_size are seeded from the outputs of the nonlinear programming (NLP) solver, in order to give the population some good starting points. In this paper we utilised fmincon() routine of the Matlab software as the NLP solver and each of its outputs is mapped to a chromosome.

\subsection{Decoding procedure for PTbR}

The decoding procedure is invoked on each individual to perform a mapping from a chromosome (i.e., the encoding space) to a MCFP network instance (i.e., the solution space). As shown in Fig. 7, there are three different possible mappings between the encoding space and the solution space. PTbR follows the 1-to- $N$ mapping, where $N$ can be any number greater than 1 . But $\mathrm{PbR}$ has the property of $N$-to- 1 mapping [29].

Since the mapping between chromosome and the solution space is 1-to- $N$ for PTbR, by applying decoding procedure (Algorithm 1) $N$ times on a given chromosome, $N$ solution vectors $(\overrightarrow{\mathbf{x}})$ will be obtained. This characteristic of 


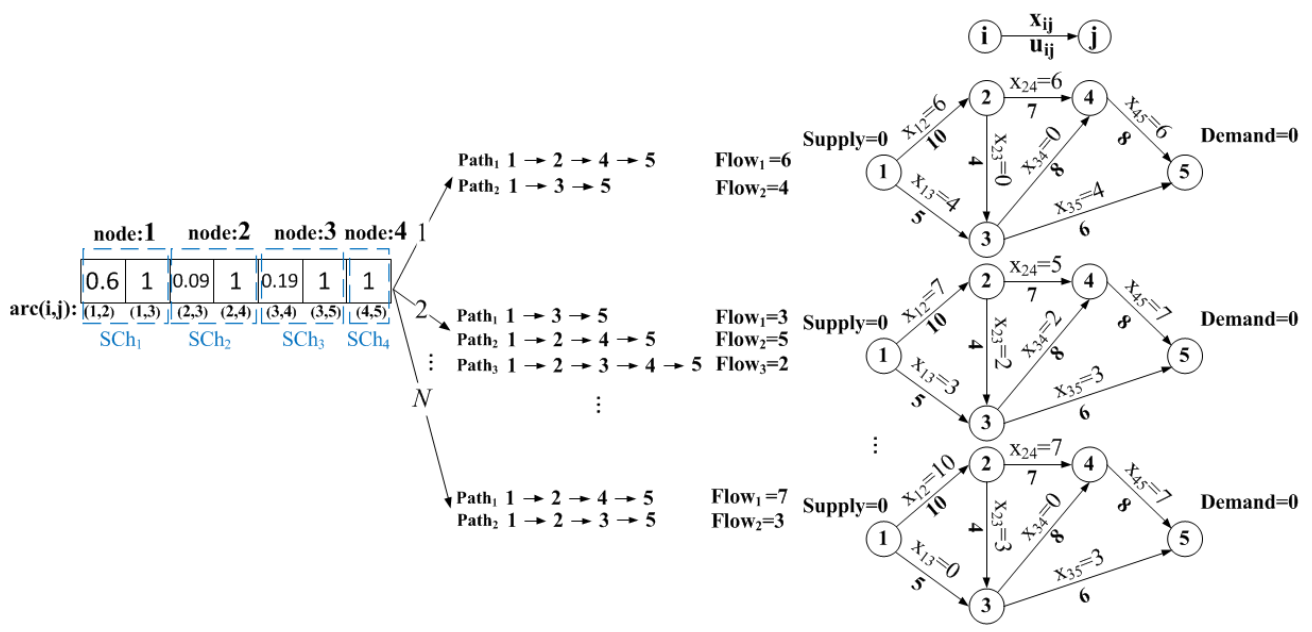

Figure 8: $N$ times decoding procedure within PTbGA for the example chromosome in Fig. 5.

the PTbR scheme improves the searching capability of PTbGA within the feasible region and provides an algorithm a better chance to generate the better quality solutions. Hence, in PTbGA, instead of performing $N=1$ time decoding for a given chromosome, the decoding procedure in Algorithm 1 is applied $N$ times for a given chromosome. The $N$ decoding procedure for the given chromosome is shown in Fig. 8.

\subsection{Crossover and mutation}

In GA, search is carried out by applying genetic operators to selected individuals from the population, e.g., using crossover and mutation operators to generate new offspring from the selected parents (chromosomes) in each generation. To perform a crossover operator, two parents are randomly selected from the population and two sub-chromosomes are randomly selected within each parent $\left(\mathrm{SCh}_{A}\right.$ and $\left.\mathrm{SCh}_{B}\right)$, as shown in Fig. 9. Then the crossover operator is applied to swap the sub-chromosomes between the two parents. To perform mutation on each chromosome, a random parent is selected from population and a randomly chosen sub-chromosome is regenerated to create a new offspring. Note that the crossover and mutation operators always produce feasible solutions. 


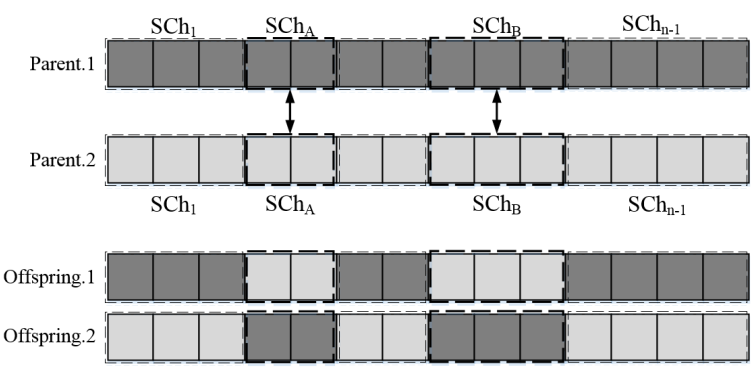

Figure 9: A two point crossover operator for PTbGA.

\subsection{Fitness evaluation and selection criteria}

As shown in Fig. 8 and for the given chromosome, the decoding procedure presented in Algorithm 1 is applied $N$ times which produces $N$ solutions in the solution space. By evaluating the $N$ solutions using Eq. 1, $N$ objective values are obtained. To evaluate the fitness value of a given chromosome, we can either: 1) calculate the average of the objective values (Ave) for the $N$ solutions; or 2) select the minimum (Best) of the $N$ objective values. Decoding $N$ times should provide more accurate information about the goodness of the chromosome than just decoding (or sampling) once. In Subsection 5.2, we demonstrate that the second approach (i.e. choosing the minimum (Best) objective value) is the most effective way of evaluating the fitness value for each chromosome. Finally, after obtaining a fitness value for each individual probabilistic tree, the tournament selection procedure is applied to select the fitter individuals from the population for the next iteration.

\subsection{Stopping criteria}

There are two stopping criteria for the PTbGA method: 1) reach the maximum number of function evaluations (NFEs); 2) no improvement in the best individual for $\beta$ successive iterations. Either of these conditions is satisfied first, the algorithm terminates and the best individual (Best_Solution) is reported as the output.

In this paper we compare the proposed PTbGA with the priority-based GA (PrGA). The PrGA method is obtained from [29] which used prioritybased representation as the encoding scheme for MCFPs. A weight mapping crossover (WMX) and inversion mutation were applied as genetic operators and tournament selection was chosen as the selection procedure $[29,33]$.

A weight mapping crossover (WMX) is proposed as an extension of onecut crossover for permutation representation [34]. To apply WMX crossover, 


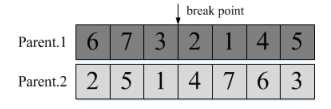

(a) Select a break point.

$$
\begin{array}{ll|l|l|l|l|l|l|l|}
\text { Parent.1 } & 6 & 7 & 3 & 4 & 7 & 6 & 3 \\
\text { Parent.2 } & 2 & 5 & 1 & 2 & 1 & 4 & 5 \\
\cline { 2 - 7 } & & & & & & & &
\end{array}
$$

(b) Swap sub-strings

\begin{tabular}{|c|c|c|c|c|c|c|c|c|c|c|}
\hline 4 & 7 & 6 & 3 & $\begin{array}{llll}3 & 4 & 6 & 7\end{array}$ & Offspring. 1 & \begin{tabular}{|l|l}
6 & 7 \\
\end{tabular} & 3 & 1 & 2 & \begin{tabular}{l|l}
4 & 5
\end{tabular} \\
\hline 2 & 1 & 4 & 5 & $\begin{array}{llll}1 & 2 & 4 & 1\end{array}$ & Offspring.2 & \begin{tabular}{|l|l}
2 & 5 \\
\end{tabular} & 1 & 3 & 4 & 67 \\
\hline
\end{tabular}
between parents. right sub-strings. spring.

Figure 10: An example of a weight mapping crossover (WMX) for PrGA.

two parents are randomly selected from the population and a random breakpoint is chosen, as shown in Fig. 10a. Then the sub-strings on the right side of the breakpoint are swapped between parents (Fig. 10b). Sub-strings located on the right side of the break point are sorted and mapping between those sub-strings is done (Fig. 10c). Finally, the offspring based on the mapping is generated as shown in Fig. 10d. To perform inversion mutation, a parent, as well as two break points within the chromosome are randomly selected. Then the sub-string between two selected break points is inverted and an offspring is generated.

\section{Experimental Studies}

In this section, we first describe the MCFP instances and cost functions that have been adopted, followed by some discussion about the mathematical solver packages used in our experiments. We then present the parameter setting, experimental result analysis on the performances of PrGA, PTbGA, and mathematical solvers in solving the MCFP instances.

Since we want to solve the MCFP instances using nonlinear non-convex functions, a set of nonlinear non-convex functions commonly-used in the literature has been adopted [35, 36, 37]. Michalewicz et al. [37] categorised these nonlinear cost functions as 1) piece-wise linear cost functions; 2) multimodal (nonlinear non-convex) cost functions; 3) smooth cost functions which are mostly used for Operations Research (OR) problems. In this paper we chose the nonlinear non-convex and arc-tangent approximation of the piecewise linear cost functions to compare the performances the PTbGA, PrGA and two mathematical solver packages. The formulation of these functions are as follows [37, 35, 36]: 


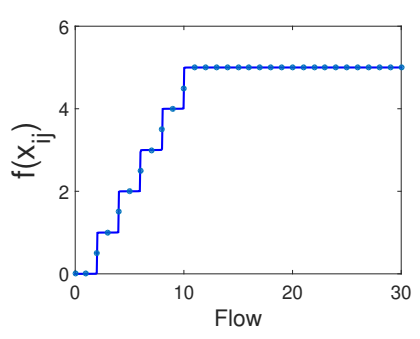

(a) $F_{1}$

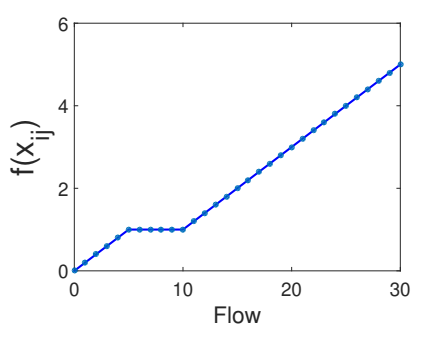

(b) $F_{2}$

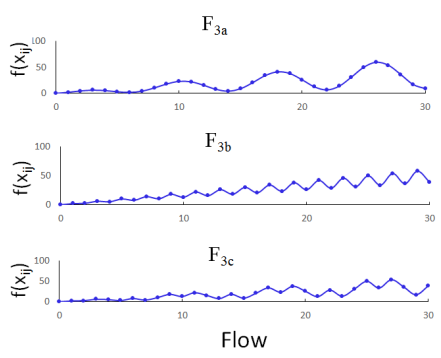

(c) $F_{3 a}, F_{3 b}$ and $F_{3 c}$

Figure 11: Shapes of different cost functions.

$$
\begin{array}{r}
F_{1}: f\left(x_{i j}\right)=c_{i j}\left(\arctan \left(P_{A}\left(x_{i j}-S\right)\right) / \pi+0.5+\right. \\
\arctan \left(P_{A}\left(x_{i j}-2 S\right)\right) / \pi+0.5+ \\
\arctan \left(P_{A}\left(x_{i j}-3 S\right)\right) / \pi+0.5+ \\
\arctan \left(P_{A}\left(x_{i j}-4 S\right)\right) / \pi+0.5+ \\
\left.\arctan \left(P_{A}\left(x_{i j}-5 S\right)\right) / \pi+0.5\right) . \\
F_{2}: f\left(x_{i j}\right)=c_{i j}\left(\left(x_{i j} / S\right)\left(\arctan \left(P_{B} x_{i j}\right) / \pi+0.5\right)+\right. \\
\left(1-x_{i j} / S\right)\left(\arctan \left(P_{B}\left(x_{i j}-S\right)\right) / \pi+0.5\right)+ \\
\left.\left(x_{i j} / S-2\right)\left(\arctan \left(P_{B}\left(x_{i j}-2 S\right)\right) / \pi+0.5\right)\right) . \\
F_{3}: f\left(x_{i j}\right)=100 \times c_{i j}\left(x_{i j}\left(\sin \left(\frac{5 \pi x_{i j}^{w}}{4 S}\right)+1.3\right)\right) .
\end{array}
$$

Note that $P_{A}$ and $P_{B}$ are set to $1000, S$ is set to 5 and $c_{i j}$ is non-negative coefficient $[14,36]$. To examine the robustness of PTbGA, the parameter $w$ in the cost function $F_{3}$ is set to 1,2 and 3 , to generate $F_{3 a}, F_{3 b}$ and $F_{3 c}$ functions, respectively. These cost functions $F_{1}$ to $F_{3}$ are illustrated in Fig. 11.

A set of 36 capacitated network instances are randomly generated with $n=\{5,10,20,40,60,80,100,150,200,250,400,500\}$ nodes and presented in Table 2, where No. denotes the instance number. Note that for each node size $n$, we generate 3 different instances with $m$ arcs. The network instances are grouped as small-sized instances (5 and 10 nodes), mediumsized instances (20-60 nodes) and large-sized instances (80-500 nodes). We are not able to utilise the existing benchmark in the literature, since all existing instances are uncapacitated networks, and the largest size of the 
Table 2: A set of 36 randomly generated network instances.

\begin{tabular}{|c|c|c|c|c|c|c|c|c|c|c|c|c|c|c|}
\hline \multicolumn{6}{|c|}{ " small-sized problems } & \multicolumn{9}{|c|}{ " medium-sized problems } \\
\hline No. & $n$ & $m$ & No. & $n$ & $m$ & No. & $n$ & $m$ & No. & $n$ & $m$ & No. & $n$ & $m$ \\
\hline 1 & \multirow{3}{*}{5} & 7 & 4 & \multirow{3}{*}{10} & 35 & 7 & \multirow{3}{*}{20} & 85 & 10 & \multirow{3}{*}{40} & 263 & 13 & \multirow{3}{*}{60} & 717 \\
\hline 2 & & 9 & 5 & & 32 & 8 & & 104 & 11 & & 374 & 14 & & 724 \\
\hline 3 & & 7 & 6 & & 24 & 9 & & 93 & 12 & & 336 & 15 & & 663 \\
\hline \multicolumn{15}{|c|}{ large-sized problems } \\
\hline No. & $n$ & $m$ & No. & $n$ & $m$ & No. & $n$ & $m$ & No. & $n$ & $m$ & No. & $n$ & $m$ \\
\hline 16 & \multirow{3}{*}{80} & 1306 & 19 & \multirow{3}{*}{100} & 1894 & 22 & \multirow{3}{*}{150} & 3770 & 25 & \multirow{3}{*}{200} & 7704 & 28 & \multirow{3}{*}{250} & 12027 \\
\hline 17 & & 1278 & 20 & & 2141 & 23 & & 4205 & 26 & & 7546 & 29 & & 12134 \\
\hline 18 & & 1235 & 21 & & 2063 & 24 & & 4590 & 27 & & 7768 & 30 & & 11771 \\
\hline No. & $n$ & $m$ & No. & $n$ & $m$ & & & & & & & & & \\
\hline 31 & \multirow{3}{*}{400} & 30247 & 34 & \multirow{3}{*}{500} & 47029 & & & & & & & & & \\
\hline 32 & & 30210 & 35 & & 47554 & & & & & & & & & \\
\hline 33 & & 29556 & 36 & & 48839 & & & & & & & & & \\
\hline
\end{tabular}

networks are 50 nodes, however in this paper we aim to solve the large-scale capacitated network instances with up to 500 nodes and 48,000 arcs.

\subsection{Mathematical programming methods and challenges}

The MCFP using nonlinear non-convex cost functions is categorised as mixed integer nonlinear programming (MINLP) problems. There are limited mathematical solver packages which are able to solve MINLPs such as CPLEX, Couenne, Baron, LINDOGlobal and AlphaECP [38, 39, 40]. However, each of these solvers has some limitations. For instance, CPLEX is only able to solve quadratic optimisation problems and it is unable to model and solve general MINLPs. BARON [41] cannot handle the trigonometric functions $\sin (x), \cos (x)$, while Couenne is not able to handle the arctangent function. Among these solvers, AlphaECP and LINDOGlobal are the only ones which are able to handle general MINLPs [39, 40]. Considering all the limitations that mathematical programming methods encountered, in this paper we choose to compare PTbGA and PrGA's results with those of LINDOGlobal and AlphaECP.

\subsection{Parameter settings}

In this subsection we study the behaviour of different parameters and operators of the PTbGA and PrGA. One way of doing that is to use a full factorial design to study the behaviour of parameters and operators [42, 43, 44]. When the number of factors increases, the full factorial design becomes inefficient [45]. The most important parameters for PTbGA are: population size 
Table 3: Factors and their levels in the proposed PTbGA.

\begin{tabular}{|c|c|c|c|c|c|c|c|c|c|c|c|c|c|c|}
\hline Factors & Symbols & $\begin{array}{ll}\text { Level } \\
\end{array}$ & Factors & Symbols & "Levels & Factors & Symbols & L Levels & Factors & Symbols & "Levels & Factors & Symbols & "Levels \\
\hline \multirow{4}{*}{$N$ decoding } & $\mathrm{A}(1)$ & 1 & \multirow{4}{*}{ pop_size } & $\mathrm{B}(1)$ & 20 & \multirow{4}{*}{$P_{c}$} & $\mathrm{C}(1)$ & 0.80 & \multirow{4}{*}{$P_{m}$} & $\mathrm{D}(1)$ & 0.10 & \multirow{4}{*}{ Fitness } & $\mathrm{E}(1)$ & Ave \\
\hline & $\mathrm{A}(2)$ & 5 & & $\mathrm{~B}(2)$ & 40 & & $\mathrm{C}(2)$ & 0.85 & & $\mathrm{D}(2)$ & 0.15 & & $\mathrm{E}(2)$ & \multirow[t]{3}{*}{ Best } \\
\hline & $\mathrm{A}(3)$ & 10 & & $\mathrm{~B}(3)$ & 60 & & $\mathrm{C}(3)$ & 0.90 & & $\mathrm{D}(3)$ & 0.20 & & & \\
\hline & $\mathrm{A}(4)$ & 20 & & $\mathrm{~B}(4)$ & 80 & & $\mathrm{C}(4)$ & 0.95 & & $\mathrm{D}(4)$ & 0.25 & & & \\
\hline
\end{tabular}

Table 4: Factors and their levels in the PrGA.

\begin{tabular}{|c|c|c|c|c|c|c|c|c|}
\hline Factors & Symbols & Levels & Factors & Symbols & Levels & Factors & Symbols & $\overline{\text { Levels }}$ \\
\hline \multirow{3}{*}{ pop_size } & $\mathrm{A}(1)$ & 100 & \multirow{3}{*}{$P_{c}$} & $\mathrm{~B}(1)$ & 0.80 & \multirow{3}{*}{$P_{m}$} & $\mathrm{C}(1)$ & 0.10 \\
\hline & $\mathrm{A}(2)$ & 200 & & $\mathrm{~B}(2)$ & 0.85 & & $\mathrm{C}(2)$ & 0.15 \\
\hline & $\mathrm{A}(3)$ & 300 & & $\mathrm{~B}(3)$ & 0.90 & & $\mathrm{C}(3)$ & 0.20 \\
\hline
\end{tabular}

(pop_size), $N$ times decoding (or sampling) for each chromosome, crossover rate $\left(P_{c}\right)$, mutation rate $\left(P_{m}\right)$. One important consideration for PTbGA is how we evaluate the fitness value of a chromosome, either using the average of $N$ objective values (Ave) or using the minimum (Best) of the $N$ objective values (Subsection 4.4). For PrGA, the most important parameters are pop_size, $P_{c}$ and $P_{m}$. These parameters for PTbGA and PrGA and their levels are presented in Tables 3 and 4 . As shown in Table 3, for PTbGA, we consider four 4-level factors and one 2-level factor with 30 network instances which runs five times [45]. Hence, the total number of runs for a full factorial design for PTbGA is $36 \times 4^{4} \times 2^{1} \times 5=92,160$, and this value for PrGA is $36 \times 3^{3} \times 5=4,860$.

Instead of running the full factorial design test, several experimental designs have been suggested to reduce the number of experiments [46]. Among these, the Taguchi method has been successfully applied for a systematic approach in optimisation [47]. Using the Taguchi design for our experiments, we try to evaluate different combinations of parameter values. We also try to maximise the performance of the signal-to-noise $(\mathrm{S} / \mathrm{N})$ ratios by solving a partial set of experiments (instead of the full set of experiments) utilising the orthogonal arrays [48]. In our experiments, the desirable value (response variable) is denoted by signal, and the undesirable (standard deviation) value is denoted by noise. The $\mathrm{S} / \mathrm{N}$ ratio of the minimisation objective is as follows [49]:

$$
S / N \quad \text { ratio }=-10 \log _{10}(\text { objective function })^{2} .
$$

In Taguchi methods, adopting the proper orthogonal array for the chosen problem can be a challenging task [50]. For PTbGA, we have four 4-level factors and one 2-level factor. Hence, the appropriate orthogonal array for 
this method is L16. In L16 $\left(4^{\wedge} 5\right)$ orthogonal array, five 4-level factors are considered. But in our PTbGA we have four 4-level and one 2-level (the last factor is fitness). To assign the 2-level factor to the 4-level column from the orthogonal array L16, each of these levels is required to be replicated twice. It is essential to notice that, after applying these techniques, the obtained array remains orthogonal and this method is called means of adjustment techniques [51]. For PrGA, since we have three 3-level factors, the appropriate orthogonal array is L9 $\left(3^{\wedge} 3\right)$.

Since we have L16 and L9 orthogonal arrays, there are 16 and 9 different combinations of control factors for PTbGA and PrGA, respectively. Both PrGA and PTbGA are implemented in MATLAB on a PC with Intel(R) Core(TM) i7-6500U $2.50 \mathrm{GHz}$ processor with 8 GB RAM. Each algorithm is evaluated on a set of 36 instances (Table 2) using cost function $F_{3 a}$ for each combination. In order to have a fair comparison, for both PTbGA and PrGA, a fixed computational budget for each trial is considered. Since the scale of an objective function value for each instance may be different, the relative percentage deviation (RPD) is used for each instance and calculated by [51]:

$$
R P D=\frac{A l g_{\text {sol }}-\text { Min }_{\text {sol }}}{\text { Min }_{\text {sol }}}
$$

where $A l g_{\text {sol }}$ and $\mathrm{Min}_{\text {sol }}$ are the obtained objective value and the best solution found for each replication of a trial over a given instance, respectively. First, we calculate the average RPD for each trial. Then, by using Eq. 8, the $\mathrm{S} / \mathrm{N}$ ratio is obtained for each trial. Finally, the $\mathrm{S} / \mathrm{N}$ ratios of trials at each level of factors are averaged and the results are shown in Figs. 12 and 13 for PTbGA and PrGA, respectively. For instance, as shown in Fig. 13, to obtain the value of -17.17 for factor $\mathrm{A}$ at level 3 (i.e. $\mathrm{A}(3)$ ), we should calculate the average of $\mathrm{S} / \mathrm{N}$ ratios for all trials in which factor $\mathrm{A}$ at level 3 . As shown in Fig. 12 and based on Table 3, the best values for factors A, B, $\mathrm{C}, \mathrm{D}$, and $\mathrm{E}$ are $2,3,1,1$, and 2 respectively. These results demonstrate that the $N=5$ times decoding, pop_size $=60, P_{c}=0.8, P_{m}=0.1$, and assigning the Best (i.e., the minimum) objective value among $N$ objective values have the highest performance for PTbGA. As shown in Fig. 13 and based on Table 4 for PrGA, the best values for A, B, and C are 3, 2, and 1 respectively (i.e. pop_size $=300, P_{c}=0.85$ and $P_{m}=0.1$ ).

The other parameters for PTbGA and PrGA are set as follows: maximum number of iterations $\left(I t_{\max }=200\right)$ and maximum number of function evalu- 


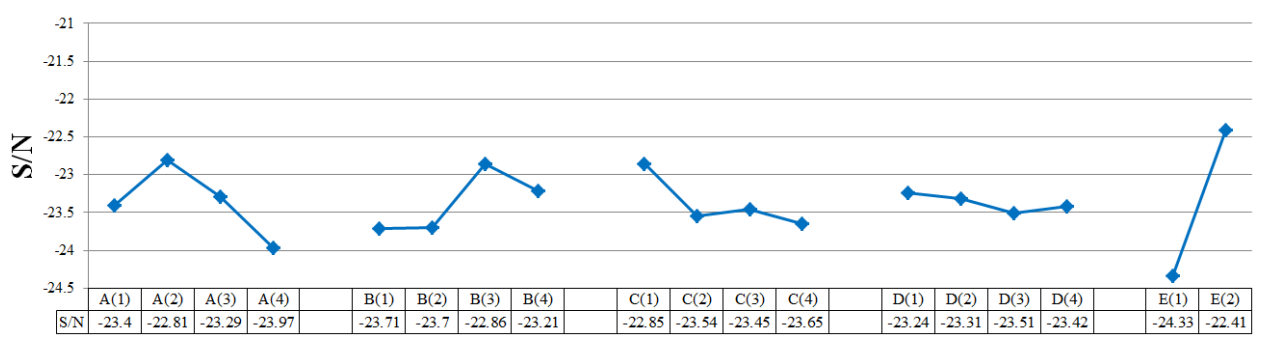

Figure 12: Mean S/N ratio plot for each level of the factors in PTbGA.

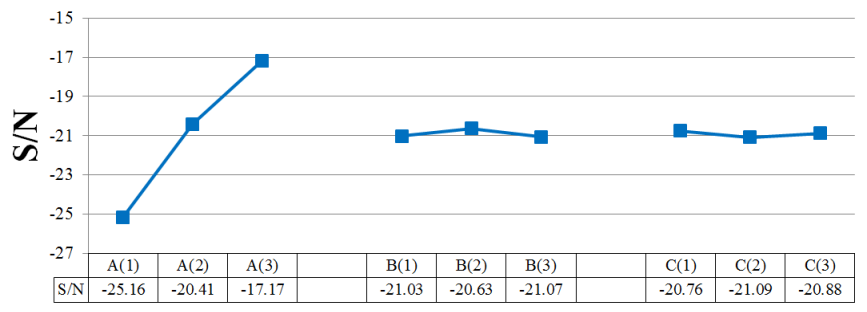

Figure 13: Mean S/N ratio plot for each level of the factors in PrGA.

ations $(N F E s=100,000)$. In order to have an adequate level of randomness in the initialisation phase, $\alpha$ is set to $95 \%$. If the results are not improved in $\beta=30$ successive iterations, the algorithm is terminated. The parameter for mathematical solvers (AlphaECP and LINDOGlobal) is running time which is set to 3600 seconds (s) for problem instances up to $n=100$ nodes and $7200 \mathrm{~s}$ for larger problem instances.

\subsection{Results and analysis}

After defining the parameters and operators for PTbGA and PrGA, we use a set of 36 network instances using cost functions $F_{1}, F_{2}, F_{3 a}, F_{3 b}$ and $F_{3 c}$ to evaluate PTbGA, PrGA, AlphaECP, and LINDOGlobal. The results are provided in Tables 5 to 9 . std and $t$ (for PTbGA and PrGA) denote the standard deviation of the results and the average of running time in seconds respectively, and the mean represents the average of objective function values over 30 runs. The $t^{\prime}$ and $O B J$ for LINDOGlobal and AlphaECP denote the running time and the objective value, respectively. "NF" denotes that the mathematical solver cannot find any feasible solution within the time limit. For LINDOGlobal and AlphaECP on network instances up to 100 nodes, the time limit is set to $3600 \mathrm{~s}$, and on instances with $n=\{150,200,250,400,500\}$, it is set to $7200 \mathrm{~s}$. In some cases the solvers find the optimal solution be- 
Table 5: Result comparison of PTbGA with other algorithms using cost function $F_{1}$.

\begin{tabular}{|c|c|c|c|c|c|c|c|c|c|c|c|c|c|c|}
\hline \multicolumn{15}{|c|}{ small-sized instances } \\
\hline \multirow{2}{*}{ No. } & \multirow{2}{*}{$n$} & \multirow{2}{*}{$m$} & \multicolumn{3}{|c|}{ PTbGA } & \multicolumn{3}{|c|}{ PrGA } & \multicolumn{2}{|c|}{ LINDOGlobal } & \multicolumn{2}{|c|}{ AlphaECP } & \multirow{2}{*}{ Gap } & \multirow{2}{*}{$h$} \\
\hline & & & $t$ & mean & std & $t$ & mean & std & $t^{\prime}$ & $O B J$ & $t^{\prime}$ & $O B J$ & & \\
\hline 1 & \multirow{3}{*}{5} & 7 & 32 & 0.0038 & $0.00 \mathrm{E}+00$ & 26 & 4.0018 & $0.00 \mathrm{E}+00$ & 1 & 0.0038 & 2 & 4.0017 & $0.0 \%$ & 0 \\
\hline 2 & & 9 & 28 & 1.0033 & $2.28 \mathrm{E}-16$ & 27 & 4.0015 & $9.11 \mathrm{E}-16$ & 2 & 1.0033 & 4 & 4.0015 & $0.0 \%$ & 0 \\
\hline 3 & & 7 & 35 & 1.0027 & $2.28 \mathrm{E}-16$ & 30 & 4.0012 & $0.00 \mathrm{E}+00$ & 1 & 1.0027 & 1 & 3.0016 & $0.0 \%$ & 0 \\
\hline 4 & \multirow{3}{*}{10} & 35 & 57 & 0.0098 & $1.26 \mathrm{E}-05$ & 65 & 4.0083 & $5.32 \mathrm{E}-05$ & 6 & 0.0098 & 55 & 2.0097 & $0.0 \%$ & 0 \\
\hline 5 & & 32 & 73 & 0.0092 & $1.47 \mathrm{E}-05$ & 65 & 2.5085 & $2.14 \mathrm{E}-04$ & 10 & 0.0092 & 17 & 3.0080 & $0.0 \%$ & 0 \\
\hline 6 & & 24 & 60 & 1.0071 & $4.56 \mathrm{E}-16$ & 66 & 3.5065 & $4.56 \mathrm{E}-16$ & 112 & 1.0071 & 250 & 2.5073 & $0.0 \%$ & 0 \\
\hline \multicolumn{15}{|c|}{ medium-sized instances } \\
\hline 7 & \multirow{3}{*}{20} & 85 & 120 & 0.0244 & $1.30 \mathrm{E}-05$ & 116 & 2.0245 & $2.69 \mathrm{E}-05$ & 3600 & 0.0243 & 150 & 0.0254 & $-0.4 \%$ & -1 \\
\hline 8 & & 104 & 130 & 0.0307 & $2.93 \mathrm{E}-06$ & 128 & 0.0311 & $0.00 \mathrm{E}+00$ & 575 & 0.0307 & 162 & 0.0308 & $0.0 \%$ & 0 \\
\hline 9 & & 93 & 95 & 1.5270 & $1.19 \mathrm{E}-05$ & 99 & 2.5276 & $2.40 \mathrm{E}-04$ & 3600 & 1.5270 & 135 & 6.5256 & $0.0 \%$ & 0 \\
\hline 10 & \multirow{3}{*}{40} & 263 & 168 & 0.0705 & $3.16 \mathrm{E}-06$ & 165 & 0.2474 & $2.44 \mathrm{E}-01$ & 3600 & 0.0704 & 3600 & 0.0707 & $-0.1 \%$ & -1 \\
\hline 11 & & 374 & 195 & 0.1036 & $4.27 \mathrm{E}-17$ & 195 & 0.1043 & $2.07 \mathrm{E}-05$ & 3600 & 0.1036 & 3600 & 0.1037 & $0.0 \%$ & 0 \\
\hline 12 & & 336 & 207 & 0.0913 & $2.85 \mathrm{E}-17$ & 197 & 0.0938 & $1.56 \mathrm{E}-04$ & 3600 & 0.0913 & 3600 & 0.0926 & $0.0 \%$ & 0 \\
\hline 13 & \multirow{3}{*}{60} & 717 & 261 & 0.2065 & $4.26 \mathrm{E}-06$ & 262 & 3.7306 & $1.12 \mathrm{E}-01$ & 3600 & 0.2065 & 3600 & 0.2079 & $0.0 \%$ & 0 \\
\hline 14 & & 724 & 273 & 0.1893 & $2.01 \mathrm{E}-05$ & 266 & 0.4662 & 4.43E-01 & 3600 & 0.1893 & 3600 & 0.1908 & $0.0 \%$ & 0 \\
\hline 15 & & 663 & 262 & 0.1874 & $5.70 \mathrm{E}-17$ & 261 & 0.214 & $1.12 \mathrm{E}-01$ & 3600 & 0.1874 & 3600 & 0.1881 & $0.0 \%$ & 0 \\
\hline \multicolumn{15}{|c|}{ large-sized instances } \\
\hline 16 & \multirow{3}{*}{80} & 1306 & 466 & 0.3469 & $1.69 \mathrm{E}-05$ & 439 & 5.2462 & $2.61 \mathrm{E}-01$ & 3600 & 0.3469 & 3600 & 0.8494 & $0.0 \%$ & 0 \\
\hline 17 & & 1278 & 450 & 0.3334 & $1.37 \mathrm{E}-05$ & 436 & 1.5602 & $3.02 \mathrm{E}-01$ & 3600 & 0.3333 & 3600 & 0.3343 & $0.0 \%$ & -1 \\
\hline 18 & & 1235 & 485 & 0.3359 & $9.48 \mathrm{E}-06$ & 462 & 1.3374 & $2.28 \mathrm{E}-04$ & 3600 & 0.3359 & 3600 & 2.3361 & $0.0 \%$ & 0 \\
\hline 19 & \multirow{3}{*}{100} & 1894 & 629 & 0.4970 & $1.39 \mathrm{E}-05$ & 620 & 0.5486 & $1.54 \mathrm{E}-01$ & 3600 & 0.4971 & 3600 & 0.4973 & $0.0 \%$ & 1 \\
\hline 20 & & 2141 & 641 & 6.0648 & $6.08 \mathrm{E}-06$ & 645 & 6.5654 & $3.08 \mathrm{E}-05$ & 3600 & 6.0648 & 3600 & 6.0649 & $0.0 \%$ & 0 \\
\hline 21 & & 2063 & 580 & 0.5561 & $1.57 \mathrm{E}-05$ & 585 & 2.5568 & $1.51 \mathrm{E}-04$ & 3600 & 0.55610 & 3600 & 0.5574 & $0.0 \%$ & 0 \\
\hline 22 & \multirow{3}{*}{150} & \begin{tabular}{|l|}
3770 \\
\end{tabular} & 1067 & 0.9845 & $2.37 \mathrm{E}-05$ & 1061 & 1.8852 & $3.08 \mathrm{E}-01$ & 7200 & $\mathrm{NF}$ & \begin{tabular}{|l}
7200 \\
\end{tabular} & 0.9851 & $0.1 \%$ & 1 \\
\hline 23 & & 4205 & 1679 & 1.112 & $1.49 \mathrm{E}-04$ & 1670 & 9.158 & $1.54 \mathrm{E}-01$ & 7200 & $\mathrm{NF}$ & 7200 & 1.6131 & $45.1 \%$ & 1 \\
\hline 24 & & 4590 & 1160 & 1.1731 & $2.28 \mathrm{E}-16$ & 1136 & 1.1738 & $1.20 \mathrm{E}-04$ & 7200 & 1.1731 & 7200 & 2.6729 & $0.0 \%$ & 0 \\
\hline 25 & \multirow{3}{*}{200} & \begin{tabular}{|l|}
7704 \\
\end{tabular} & 1703 & 2.0237 & $1.91 \mathrm{E}-05$ & 1698 & 2.0253 & $1.64 \mathrm{E}-04$ & 7200 & $\mathrm{NF}$ & 7200 & 2.0242 & $0.0 \%$ & 1 \\
\hline 26 & & 7546 & 1683 & 1.9875 & $2.20 \mathrm{E}-04$ & 1657 & 6.8599 & 7.93E-01 & 7200 & $\mathrm{NF}$ & 7200 & 3.4853 & $75.4 \%$ & 1 \\
\hline 27 & & 7768 & 1781 & 1.9900 & $6.68 \mathrm{E}-05$ & 1770 & 9.8616 & $2.75 \mathrm{E}-01$ & 7200 & $\mathrm{NF}$ & 7200 & 3.9902 & $100.5 \%$ & 1 \\
\hline 28 & & \begin{tabular}{|l}
12027 \\
\end{tabular} & 2680 & 3.2414 & $1.24 \mathrm{E}-04$ & 2666 & 3.2418 & $1.64 \mathrm{E}-04$ & 7200 & $\mathrm{NF}$ & \begin{tabular}{|l}
7200 \\
7200
\end{tabular} & 3.2417 & $0.0 \%$ & 1 \\
\hline 29 & 250 & 12134 & 2632 & 3.1387 & $5.52 \mathrm{E}-04$ & 2617 & 7.1371 & $1.03 \mathrm{E}-04$ & 7200 & $\mathrm{NF}$ & 7200 & 4.1395 & $31.9 \%$ & 1 \\
\hline 30 & & 11771 & 2419 & 3.0327 & $4.68 \mathrm{E}-16$ & 2391 & 3.5352 & $5.29 \mathrm{E}-04$ & 7200 & NF & 7200 & 3.0327 & $0.0 \%$ & 0 \\
\hline 31 & & \begin{tabular}{|l}
30247 \\
\end{tabular} & 3048 & 11.2415 & $1.29 \mathrm{E}-01$ & 2959 & 23.7160 & $2.86 \mathrm{E}+00$ & 7200 & $\mathrm{NF}$ & \begin{tabular}{|l}
7200 \\
7200
\end{tabular} & 26.7962 & $138.4 \%$ & 1 \\
\hline 32 & 400 & 30210 & 3062 & 7.8540 & $6.99 \mathrm{E}-13$ & 3035 & 13.1003 & $3.53 \mathrm{E}-01$ & 7200 & $\mathrm{NF}$ & 7200 & 13.8495 & $76.3 \%$ & 1 \\
\hline 33 & & 29556 & 2969 & 7.6520 & $9.11 \mathrm{E}-16$ & 3037 & 9.6828 & $1.29 \mathrm{E}-01$ & 7200 & $\mathrm{NF}$ & 7200 & 10.1493 & $32.6 \%$ & 1 \\
\hline 34 & & \begin{tabular}{|l}
47029 \\
\end{tabular} & 3512 & 12.1615 & $1.12 \mathrm{E}-04$ & 3485 & 14.6596 & $2.55 \mathrm{E}-04$ & 7200 & $\mathrm{NF}$ & \begin{tabular}{|l|}
7200 \\
\end{tabular} & 14.6602 & $20.5 \%$ & 1 \\
\hline 35 & 500 & 47554 & 3561 & 12.5239 & $2.62 \mathrm{E}-04$ & 3588 & 12.5242 & $7.15 \mathrm{E}-05$ & 7200 & $\mathrm{NF}$ & 7200 & 32.5245 & $159.7 \%$ & 1 \\
\hline 36 & & 48839 & 3471 & 12.8661 & $1.30 \mathrm{E}-04$ & 3479 & 14.6661 & $3.16 \mathrm{E}-01$ & 7200 & $\mathrm{NF}$ & 7200 & 15.3649 & $19.4 \%$ & 1 \\
\hline
\end{tabular}

fore reaching the time limit or other stopping criterion is met (e.g., on NLP sub-problems it stops improving), and consequently the algorithms are terminated.

In order to compare the performance of PTbGA with PrGA (two GA variants method), the $t$-test with the significance level of 0.05 is performed. The PTbGA outperforms PrGA in all instances using different cost functions $\left(F_{1}-F_{3 c}\right)$. It is clearly evident that the superior performance of the PTbGA comes from utilising PTbR in its procedure, instead of using PbR in PrGA. For instance, in Table 5, PrGA cannot find optimal solutions for all small instances (No.1-6) using function $F_{1}$. It is apparent that $\mathrm{PbR}$ is restricted 
Table 6: Result comparison of PTbGA with other algorithms using cost function $F_{2}$.

\begin{tabular}{|c|c|c|c|c|c|c|c|c|c|c|c|c|c|c|}
\hline \multicolumn{15}{|c|}{ small-sized instances } \\
\hline \multirow{2}{*}{ No. } & \multirow{2}{*}{$n$} & \multirow{2}{*}{$m$} & \multicolumn{3}{|c|}{ PTbGA } & \multicolumn{3}{|c|}{ PrGA } & \multicolumn{2}{|c|}{ LINDOGlobal } & \multicolumn{2}{|c|}{ AlphaECP } & \multirow{2}{*}{ Gap } & \multirow{2}{*}{$h$} \\
\hline & & & $t$ & mean & std & $t$ & mean & std & $t^{\prime}$ & $O B J$ & $t^{\prime}$ & $O B J$ & & \\
\hline 1 & \multirow{3}{*}{5} & 7 & 24 & 3.9997 & $4.56 \mathrm{E}-16$ & 25 & 3.9997 & $9.11 \mathrm{E}-16$ & 1 & 3.9997 & 2 & 3.9997 & $0.0 \%$ & 0 \\
\hline 2 & & 9 & 31 & 3.9997 & $0.00 \mathrm{E}+00$ & 33 & 3.9997 & $9.11 \mathrm{E}-16$ & 9 & 3.9997 & 2 & 3.9997 & $0.0 \%$ & 0 \\
\hline 3 & & 7 & 31 & 3.9996 & $4.56 \mathrm{E}-16$ & 28 & 3.9997 & $0.00 \mathrm{E}+00$ & 2 & 3.9996 & 1 & 3.9996 & $0.0 \%$ & 0 \\
\hline 4 & \multirow{3}{*}{10} & 35 & 80 & 5.9992 & $1.82 \mathrm{E}-15$ & 82 & 6.1994 & $2.73 \mathrm{E}-15$ & 631 & 5.9992 & 39 & 7.1996 & $0.0 \%$ & 0 \\
\hline 5 & & 32 & 56 & 6.7994 & $9.11 \mathrm{E}-16$ & 61 & 6.7994 & $9.11 \mathrm{E}-16$ & 325 & 6.7994 & 28 & 8.9991 & $0.0 \%$ & 0 \\
\hline 6 & & 24 & 69 & 7.7994 & $4.56 \mathrm{E}-15$ & 65 & 7.7995 & $9.11 \mathrm{E}-16$ & 246 & 7.7994 & 21 & 7.7995 & $0.0 \%$ & 0 \\
\hline \multicolumn{15}{|c|}{ medium-sized instances } \\
\hline 7 & \multirow{3}{*}{20} & 85 & 127 & 9.1987 & $3.65 \mathrm{E}-15$ & 124 & 9.399 & $3.65 \mathrm{E}-15$ & 3600 & 9.1987 & 85 & 12.7981 & $0.0 \%$ & 0 \\
\hline 8 & & 104 & 106 & 8.5986 & 3.65E-15 & 115 & 8.5989 & $2.85 \mathrm{E}-05$ & 3600 & 8.5986 & 142 & 8.5986 & $0.0 \%$ & 0 \\
\hline 9 & & 93 & 252 & 10.7987 & $1.82 \mathrm{E}-15$ & 251 & 11.8288 & 7.32E-02 & 3600 & 10.7987 & 123 & 13.5980 & $0.0 \%$ & 0 \\
\hline 10 & \multirow{3}{*}{40} & 263 & 170 & 9.7988 & $1.15 \mathrm{E}-04$ & 163 & 9.9389 & $1.31 \mathrm{E}-01$ & 3600 & 10.1988 & 3600 & 10.5986 & $4.1 \%$ & 1 \\
\hline 11 & & 374 & 199 & 6.5987 & $9.11 \mathrm{E}-16$ & 208 & 7.7488 & $4.89 \mathrm{~F}$ & 3600 & 6.5987 & 3600 & 6.7990 & $0.0 \%$ & 0 \\
\hline 12 & & 336 & 183 & 8.9993 & $0.00 \mathrm{E}+00$ & 181 & 10.009 & $2.86 \mathrm{E}-01$ & 3600 & 8.9993 & 3600 & 10.3982 & $0.0 \%$ & 0 \\
\hline 13 & \multirow{3}{*}{60} & 717 & 274 & 9.7990 & $3.07 \mathrm{E}-13$ & 270 & 10.7987 & $5.99 \mathrm{E}-05$ & 3600 & 9.7990 & 3600 & 20.9981 & $0.0 \%$ & 0 \\
\hline 14 & & 724 & 254 & 10.8687 & $1.40 \mathrm{E}-02$ & 258 & 10.8687 & $3.13 \mathrm{E}-01$ & 3600 & 11.1985 & 3600 & 14.3985 & $3.0 \%$ & 1 \\
\hline 15 & & 663 & 244 & 5.8390 & 1.39E-01 & 242 & 5.9992 & $3.84 \mathrm{E}-01$ & 3600 & 6.5988 & 3600 & 9.7985 & $13.0 \%$ & 1 \\
\hline \multicolumn{15}{|c|}{ large-sized instances } \\
\hline 16 & \multirow{3}{*}{80} & 1306 & 414 & 10.5985 & $0.00 \mathrm{E}+00$ & 411 & 11.9187 & 7.69E-01 & 3600 & 12.5983 & 3600 & 10.5976 & $0.0 \%$ & -1 \\
\hline 17 & & 1278 & 413 & 12.398 & $1.82 \mathrm{E}-15$ & 422 & 12.6084 & $2.94 \mathrm{E}-01$ & 3600 & 12.3980 & 3600 & 12.5981 & $0.0 \%$ & 0 \\
\hline 18 & & 1235 & 413 & 12.6084 & $5.21 \mathrm{E}-01$ & 413 & 13.0787 & $2.93 \mathrm{E}-01$ & 3600 & 13.3985 & 3600 & 13.1984 & $4.7 \%$ & 1 \\
\hline 19 & \multirow{3}{*}{100} & 1894 & 783 & 8.1987 & $4.27 \mathrm{E}-05$ & 527 & 9.4386 & $3.59 \mathrm{E}-01$ & 3600 & 9.1983 & 3600 & 9.1984 & $12.2 \%$ & 1 \\
\hline 20 & & 2141 & 679 & 17.9483 & $7.85 \mathrm{E}-03$ & 552 & 17.9483 & $8.89 \mathrm{E}-02$ & 3600 & 17.9979 & 3600 & 19.3974 & $0.3 \%$ & 1 \\
\hline 21 & & 2063 & 677 & 15.4181 & $6.33 \mathrm{E}-02$ & 536 & 15.4385 & $3.08 \mathrm{E}-01$ & 3600 & 15.7970 & 3600 & 17.5980 & $2.5 \%$ & 1 \\
\hline 22 & \multirow{3}{*}{150} & 3770 & 1033 & 9.3986 & $7.18 \mathrm{E}-05$ & 982 & 10.1088 & $3.92 \mathrm{E}-01$ & 3600 & 9.3987 & 7200 & 13.3987 & $0.0 \%$ & 1 \\
\hline 23 & & 4205 & 1124 & 15.1481 & $2.23 \mathrm{E}-01$ & 937 & 15.3981 & $6.09 \mathrm{E}-01$ & 3600 & 16.7982 & 7200 & 15.1981 & $0.3 \%$ & 0 \\
\hline 24 & & 4590 & 1088 & 7.6291 & 4.37E-01 & 1314 & 8.3985 & $3.12 \mathrm{E}-05$ & 3600 & 8.3985 & 7200 & 8.3983 & $10.1 \%$ & 1 \\
\hline 25 & \multirow{3}{*}{200} & 7704 & 1415 & 10.3988 & $5.47 \mathrm{E}-15$ & 1585 & 10.8088 & $6.31 \mathrm{E}-01$ & 7200 & 10.3988 & 7200 & 10.9985 & $0.0 \%$ & 0 \\
\hline 26 & & 7546 & 1821 & 13.7981 & $8.54 \mathrm{E}-05$ & 1487 & 15.2389 & $1.26 \mathrm{E}+00$ & 7200 & NF & 7200 & 13.9978 & $1.4 \%$ & 1 \\
\hline 27 & & 7768 & 1439 & 15.7282 & $2.62 \mathrm{E}-01$ & 1587 & 17.4483 & $5.65 \mathrm{E}-01$ & 7200 & NF & 7200 & 17.1976 & $9.3 \%$ & 1 \\
\hline 28 & & 12027 & 2394 & 13.0486 & $5.06 \mathrm{E}-01$ & 2543 & 13.1978 & $1.54 \mathrm{E}-04$ & 7200 & $\mathrm{NF}$ & 7200 & 13.5966 & $4.2 \%$ & 1 \\
\hline 29 & 250 & 12134 & 2292 & 15.7986 & $5.03 \mathrm{E}-01$ & 2294 & 16.3182 & $1.93 \mathrm{E}-01$ & 7200 & $\mathrm{NF}$ & 7200 & 17.5968 & $11.4 \%$ & 1 \\
\hline 30 & & 11771 & 2861 & 9.3980 & $6.23 \mathrm{E}-05$ & 3083 & 10.2088 & $6.27 \mathrm{E}-01$ & 7200 & $\mathrm{NF}$ & 7200 & 9.5979 & $2.1 \%$ & 1 \\
\hline 31 & & 30247 & 3105 & 11.5452 & $3.58 \mathrm{E}-01$ & 3197 & 24.9752 & $4.37 \mathrm{E}+00$ & 7200 & NF & 7200 & 26.39478 & $128.6 \%$ & 1 \\
\hline 32 & 400 & 30210 & 3097 & 17.4518 & $1.37 \mathrm{E}+00$ & 2998 & 34.054 & $5.06 \mathrm{E}+00$ & 7200 & NF & 7200 & 37.792297 & $116.6 \%$ & 1 \\
\hline 33 & & 29556 & 3096 & 11.0387 & $8.18 \mathrm{E}-01$ & 3146 & 21.0264 & $4.40 \mathrm{E}+00$ & 7200 & NF & 7200 & 23.596053 & $113.8 \%$ & 1 \\
\hline 34 & & 47029 & 3573 & 14.0783 & $4.59 \mathrm{E}-01$ & 3513 & 23.5957 & $3.65 \mathrm{E}-15$ & 7200 & $\mathrm{NF}$ & 7200 & 23.595671 & $67.6 \%$ & 1 \\
\hline 35 & 500 & 47554 & 3418 & 8.7857 & 8.19E-01 & 3475 & 22.5954 & $8.29 \mathrm{E}-10$ & 7200 & NF & 7200 & 22.595416 & $157.2 \%$ & 1 \\
\hline 36 & & 48839 & 3493 & 12.025 & $4.46 \mathrm{E}-01$ & 3577 & 22.5954 & $1.84 \mathrm{E}-08$ & 7200 & $\mathrm{NF}$ & 7200 & 22.595416 & $87.9 \%$ & 1 \\
\hline
\end{tabular}

to a limited subset of the feasible region. As a result PrGA could not find optimal solutions for the small-sized instances.

We also compare the performance of PTbGA with LINDOGlobal and AlphaECP by performing a one-sample $t$-test with the significance level set to 0.05. If PTbGA has statistically better or worse performance than that of the mathematical solvers, the parameter $h$ is set to 1 and -1 respectively, otherwise $h$ is set to 0. To calculate the value of Gap between PTbGA and other mathematical solvers' results, the following equation is used: Gap = $\left(\left(\min \left\{O B J_{A l p h a E C P}, O B J_{L I N D O G l o b a l}\right\}-\right.\right.$ mean $\left._{P T b G A}\right) /$ mean $\left._{P T b G A}\right) \times 100$. In Tables 5-9, the best objective values among LINDOGlobal, AlphaECP and the average of 30 runs for the PTbGA and PrGA are presented in boldface. 
Table 7: Result comparison of PTbGA with other algorithms using cost function $F_{3 a}$.

\begin{tabular}{|c|c|c|c|c|c|c|c|c|c|c|c|c|c|c|}
\hline \multicolumn{15}{|c|}{ small-sized instances } \\
\hline \multirow{2}{*}{ No. } & \multirow{2}{*}{$n$} & \multirow{2}{*}{$m$} & \multicolumn{3}{|c|}{ PTbGA } & \multicolumn{3}{|c|}{ PrGA } & \multicolumn{2}{|c|}{ LINDOGlobal } & \multicolumn{2}{|c|}{ AlphaECP } & \multirow{2}{*}{ Gap } & \multirow{2}{*}{$h$} \\
\hline & & & $t$ & mean & std & $t$ & mean & $s t d$ & $t^{\prime}$ & $O B J$ & $t^{\prime}$ & $O B J$ & & \\
\hline 1 & \multirow{3}{*}{5} & 7 & 24 & 12.4142 & $3.65 \mathrm{E}-15$ & 25 & 12.4142 & $3.65 \mathrm{E}-15$ & 1 & 12.4142 & 1 & 17.7868 & $0.0 \%$ & 0 \\
\hline 2 & & 9 & 31 & 12.4142 & $3.65 \mathrm{E}-15$ & 30 & 16.6142 & $0.00 \mathrm{E}+00$ & 1 & 12.4142 & 2 & 17.7868 & $0.0 \%$ & 0 \\
\hline 3 & & 7 & 32 & 12.4142 & $3.65 \mathrm{E}-15$ & 27 & 12.4142 & $3.65 \mathrm{E}-15$ & 1 & 12.4142 & 1 & 12.4142 & $0.0 \%$ & 0 \\
\hline 4 & \multirow{3}{*}{10} & 35 & 62 & 23.3427 & $3.74 \mathrm{E}-01$ & 60 & 23.7017 & $3.23 \mathrm{E}-01$ & 578 & 22.6355 & 112 & 29.5655 & $-3.0 \%$ & -1 \\
\hline 5 & & 32 & 63 & 33.7005 & $1.46 \mathrm{E}-14$ & 62 & 39.0863 & $0.00 \mathrm{E}+00$ & 498 & 33.2152 & 119 & 48.0589 & $1.4 \%$ & -1 \\
\hline 6 & & 24 & 56 & 24.5503 & $7.29 \mathrm{E}-15$ & 59 & 52.865 & $1.46 \mathrm{E}-14$ & 147 & 24.5503 & 134 & 49.9939 & $0.0 \%$ & 0 \\
\hline \multicolumn{15}{|c|}{ medium-sized instances } \\
\hline 7 & \multirow{3}{*}{20} & 85 & 173 & 49.9574 & $0.00 \mathrm{E}+00$ & 105 & 56.8499 & $4.43 \mathrm{E}-01$ & 3600 & 49.9574 & 3600 & 96.5076 & $0.0 \%$ & 0 \\
\hline 8 & & 104 & 81 & 50.0995 & $0.00 \mathrm{E}+00$ & 101 & 51.0446 & $1.46 \mathrm{E}+00$ & 3600 & 50.0995 & 3600 & 69.2579 & $0.0 \%$ & 0 \\
\hline 9 & & 93 & 104 & 53.8000 & $2.19 \mathrm{E}-14$ & 117 & 54.2162 & $0.00 \mathrm{E}+00$ & 3600 & 53.8000 & 3600 & 55.5370 & $0.0 \%$ & 0 \\
\hline 10 & \multirow{3}{*}{40} & 263 & 165 & 43.9655 & $7.29 \mathrm{E}-15$ & 149 & 51.2448 & $6.86 \mathrm{E}-01$ & 3600 & 43.9655 & 3600 & 85.9451 & $0.0 \%$ & 0 \\
\hline 11 & & 374 & 191 & 40.1289 & $7.29 \mathrm{E}-15$ & 175 & 44.2344 & $5.34 \mathrm{E}-01$ & 3600 & 40.1289 & 3600 & 126.8589 & $0.0 \%$ & 0 \\
\hline 12 & & 336 & 108 & 48.0355 & $1.50 \mathrm{E}-14$ & 161 & 61.9575 & $2.58 \mathrm{E}+00$ & 3600 & 48.0360 & 3600 & 75.4873 & $0.0 \%$ & 1 \\
\hline 13 & \multirow{3}{*}{60} & 717 & 251 & 54.0853 & $1.46 \mathrm{E}-14$ & 245 & 61.9999 & $4.62 \mathrm{E}-01$ & 3600 & 54.0853 & 3600 & 128.8355 & $0.0 \%$ & 0 \\
\hline 14 & & 724 & 203 & 50.5289 & $1.46 \mathrm{E}-14$ & 242 & 71.3269 & $4.62 \mathrm{E}+00$ & 3600 & 50.5289 & 3600 & 84.7289 & $0.0 \%$ & 0 \\
\hline 15 & & 663 & 265 & 40.3431 & $1.46 \mathrm{E}-14$ & 232 & 52.5299 & $2.40 \mathrm{E}+00$ & 3600 & 40.3432 & 3600 & 62.9218 & $0.0 \%$ & 1 \\
\hline \multicolumn{15}{|c|}{ large-sized instances } \\
\hline 16 & \multirow{3}{*}{80} & 1306 & 404 & 53.9005 & $7.29 \mathrm{E}-15$ & 405 & 70.2128 & $3.93 \mathrm{E}+00$ & 3600 & 53.9005 & 3600 & 116.4883 & $0.0 \%$ & 0 \\
\hline 17 & & 1278 & 397 & 59.5147 & $1.46 \mathrm{E}-14$ & 359 & 68.4995 & $4.48 \mathrm{E}+00$ & 3600 & 59.5147 & 3600 & 115.0995 & $0.0 \%$ & 0 \\
\hline 18 & & 1235 & 362 & 61.0782 & $7.29 \mathrm{E}-15$ & 385 & 71.9623 & $3.83 \mathrm{E}+00$ & 3600 & 64.6284 & 3600 & 66.8711 & $5.8 \%$ & 1 \\
\hline 19 & \multirow{3}{*}{100} & 1894 & 584 & 54.0711 & $2.92 \mathrm{E}-14$ & 501 & 70.6492 & $2.74 \mathrm{E}+00$ & 3600 & 54.0711 & 3600 & 163.9421 & $0.0 \%$ & 0 \\
\hline 20 & & 2141 & 626 & 81.2045 & $3.43 \mathrm{E}-01$ & 531 & 85.2692 & $2.71 \mathrm{E}+00$ & 3600 & 79.5005 & 3600 & 137.5025 & $-2.1 \%$ & -1 \\
\hline 21 & & 2063 & 534 & 81.1964 & $3.38 \mathrm{E}-01$ & 541 & 84.3428 & $4.36 \mathrm{E}+00$ & 3600 & 81.2721 & 3600 & 114.8010 & $1.0 \%$ & 0 \\
\hline 22 & \multirow{3}{*}{150} & 3770 & 985 & 59.2212 & $4.50 \mathrm{E}+00$ & 1019 & 87.8434 & $3.30 \mathrm{E}+00$ & 7200 & 91.0721 & 7200 & 112.6650 & $53.8 \%$ & 1 \\
\hline 23 & & 4205 & 948 & 59.9465 & $2.40 \mathrm{E}+00$ & 1071 & 91.9019 & $3.66 \mathrm{E}+00$ & 7200 & 148.8142 & 7200 & 95.3878 & $59.1 \%$ & 1 \\
\hline 24 & & 4590 & 1036 & 69.7926 & $2.52 \mathrm{E}+00$ & 994 & 87.3404 & $1.75 \mathrm{E}+00$ & 7200 & 96.7432 & 7200 & 91.5421 & $31.2 \%$ & 1 \\
\hline 25 & \multirow{3}{*}{200} & 7704 & 1505 & 74.7676 & $3.49 \mathrm{E}+00$ & 1407 & 104.8014 & $2.65 \mathrm{E}+00$ & 7200 & 178.8802 & 7200 & 110.0944 & $47.2 \%$ & 1 \\
\hline 26 & & 7546 & 1418 & 86.943 & $9.33 \mathrm{E}+00$ & 1533 & 120.7734 & $1.33 \mathrm{E}+01$ & 7200 & $\mathrm{NF}$ & 7200 & 251.8740 & $189.7 \%$ & 1 \\
\hline 27 & & 7768 & 1513 & 78.9748 & $3.50 \mathrm{E}+00$ & 1648 & 86.3431 & $1.46 \mathrm{E}-14$ & 7200 & 86.3432 & 7200 & 151.3330 & $9.3 \%$ & 1 \\
\hline 28 & & 12027 & 2361 & 97.4219 & $6.13 \mathrm{E}+00$ & 2119 & 118.1286 & $6.66 \mathrm{E}+00$ & 7200 & $\mathrm{NF}$ & 7200 & 318.8519 & $227.3 \%$ & 1 \\
\hline 29 & 250 & 12134 & 2064 & 88.5444 & $5.63 \mathrm{E}+00$ & 2015 & 107.5992 & $1.54 \mathrm{E}+00$ & 7200 & $\mathrm{NF}$ & 7200 & 110.4081 & $24.7 \%$ & 1 \\
\hline 30 & & 11771 & 2096 & 71.4761 & $5.99 \mathrm{E}+00$ & 2054 & 78.0109 & $2.61 \mathrm{E}+00$ & 7200 & 104.9594 & 7200 & 86.1289 & $20.5 \%$ & 1 \\
\hline 31 & & 30247 & 3058 & 65.5005 & $4.88 \mathrm{E}+00$ & 3112 & 158.7264 & $4.51 \mathrm{E}+01$ & 7200 & NF & 7200 & 181.95736 & $177.8 \%$ & 1 \\
\hline 32 & 400 & 30210 & 2950 & 105.9835 & $6.95 \mathrm{E}+00$ & 3022 & 282.7109 & $8.75 \mathrm{E}+01$ & 7200 & $\mathrm{NF}$ & 7200 & 327.53503 & $209.0 \%$ & 1 \\
\hline 33 & & 29556 & 3026 & 59.4162 & $4.60 \mathrm{E}+00$ & 3110 & 160.9017 & $5.79 \mathrm{E}+01$ & 7200 & NF & 7200 & 203.03755 & $241.7 \%$ & 1 \\
\hline 34 & & 47029 & 3597 & 72.6626 & $4.29 \mathrm{E}+00$ & 3543 & 186.7592 & $6.21 \mathrm{E}+00$ & 7200 & $\mathrm{NF}$ & 7200 & 189.78478 & $161.2 \%$ & 1 \\
\hline 35 & 500 & 47554 & 3528 & 63.7279 & $5.24 \mathrm{E}+00$ & 3592 & 163.8156 & $4.65 \mathrm{E}+00$ & 7200 & NF & 7200 & 166.08019 & $160.6 \%$ & 1 \\
\hline 36 & & 48839 & 3415 & 61.8586 & $3.07 \mathrm{E}+00$ & 3432 & 144.5961 & $6.73 \mathrm{E}+00$ & 7200 & NF & 7200 & 147.87511 & $139.1 \%$ & 1 \\
\hline
\end{tabular}

Table 10 summarises the results of PTbGA, LINDOGlobal and AlphaECP for solving all instances of different sizes using all nonlinear non-convex cost functions. We count the number of "win-draw-lose" (W-D-L) among these methods. For each instance in the table (i.e., each row), if the value of $h$ is 1,0 or -1 , we add the number of wins, draws and loses, respectively. For instance, as shown in Table 5 and for large-sized problems, the W-D-L score is 14-6-1, meaning that PTbGA wins 14 times, draws 6 times, and loses 1 time for solving the large-sized instances using cost function $F_{1}$ as compared with the minimum of the LINDOGlobal and AlphaECP objective values. The aforementioned calculation is considered for all the results in Tables 5-9, and summarised in Table 10. 
Table 8: Result comparison of PTbGA with other algorithms using cost function $F_{3 b}$.

\begin{tabular}{|c|c|c|c|c|c|c|c|c|c|c|c|c|c|c|}
\hline \multicolumn{15}{|c|}{ small-sized instances } \\
\hline \multirow{2}{*}{ No. } & \multirow{2}{*}{$n$} & \multirow{2}{*}{$m$} & \multicolumn{3}{|c|}{ PTbGA } & \multicolumn{3}{|c|}{ PrGA } & \multicolumn{2}{|c|}{ LINDOGlobal } & \multicolumn{2}{|c|}{ AlphaECP } & \multirow{2}{*}{ Gap } & \multirow[t]{2}{*}{$h$} \\
\hline & & & $t$ & mean & std & $t$ & mean & std & $t^{\prime}$ & $O B J$ & $t^{\prime}$ & $O B J$ & & \\
\hline 1 & \multirow{3}{*}{5} & \begin{tabular}{|l|}
7 \\
\end{tabular} & 36 & 40.4142 & $0.00 \mathrm{E}+00$ & 25 & 40.4142 & $0.00 \mathrm{E}+00$ & 84 & 40.4142 & 156 & 49.2639 & $0.0 \%$ & 0 \\
\hline 2 & & 9 & 23 & 40.4142 & $0.00 \mathrm{E}+00$ & 26 & 43.2426 & $1.46 \mathrm{E}-14$ & 480 & 40.4142 & 395 & 57.7492 & $0.0 \%$ & 0 \\
\hline 3 & & 7 & 24 & 40.4142 & $0.00 \mathrm{E}+00$ & 26 & 40.4142 & $0.00 \mathrm{E}+00$ & 106 & 40.4142 & 85 & 40.4142 & $0.0 \%$ & 0 \\
\hline 4 & \multirow{3}{*}{10} & 35 & 50 & 58.0213 & $1.31 \mathrm{E}-14$ & 59 & 63.7826 & $8.80 \mathrm{E}-01$ & 3600 & 58.0213 & 3600 & 78.2284 & $0.0 \%$ & 0 \\
\hline 5 & & 32 & 63 & 63.8142 & $7.29 \mathrm{E}-15$ & 60 & 71.3596 & $5.09 \mathrm{E}+00$ & 3600 & 63.8142 & 3600 & 96.7056 & $0.0 \%$ & 0 \\
\hline 6 & & 24 & 43 & 69.8355 & $1.56 \mathrm{E}-14$ & 55 & 85.7563 & $0.00 \mathrm{E}+00$ & 3600 & 69.8355 & 3600 & 72.6640 & $0.0 \%$ & 0 \\
\hline \multicolumn{15}{|c|}{ medium-sized instances } \\
\hline 7 & \multirow{3}{*}{20} & 85 & 126 & 75.8569 & $2.44 \mathrm{E}-14$ & 112 & 90.135 & $2.92 \mathrm{E}-14$ & 3600 & 75.6284 & 3600 & 110.1137 & $-0.3 \%$ & -1 \\
\hline 8 & & 104 & 151 & 67.2292 & $1.52 \mathrm{E}+00$ & 102 & 67.9208 & $0.00 \mathrm{E}+00$ & 3600 & 65.4569 & 3600 & 83.3848 & $-2.6 \%$ & -1 \\
\hline 9 & & 93 & 97 & 97.1268 & $1.48 \mathrm{E}+00$ & 119 & 114.9492 & $1.84 \mathrm{E}-14$ & 3600 & 96.0640 & 3600 & 174.0639 & $-1.1 \%$ & -1 \\
\hline 10 & \multirow{3}{*}{40} & 263 & 243 & 77.0225 & $5.20 \mathrm{E}-01$ & 174 & 87.7858 & $1.48 \mathrm{E}+00$ & 3600 & 74.4426 & 3600 & 132.6487 & $-3.3 \%$ & -1 \\
\hline 11 & & 374 & 223 & 47.0695 & $1.84 \mathrm{E}-01$ & 188 & 59.9811 & $1.64 \mathrm{E}+00$ & 3600 & 49.0355 & 3600 & 74.5350 & $4.2 \%$ & 1 \\
\hline 12 & & 336 & 201 & 74.917 & $1.35 \mathrm{E}+00$ & 180 & 84.4553 & $4.95 \mathrm{E}+00$ & 3600 & 75.6284 & 3600 & 211.2853 & $0.9 \%$ & 1 \\
\hline 13 & \multirow{3}{*}{60} & \begin{tabular}{|l|}
717 \\
\end{tabular} & 328 & 86.9664 & $2.76 \mathrm{E}-01$ & 256 & 104.2283 & $1.06 \mathrm{E}+00$ & 3600 & 88.7208 & 3600 & 120.3770 & $2.0 \%$ & 1 \\
\hline 14 & & 724 & 326 & 81.6177 & 1.411 & 288 & 90.2898 & $2.19 \mathrm{E}+00$ & 3600 & 84.4782 & 3600 & 125.9317 & $3.5 \%$ & 1 \\
\hline 15 & & 663 & 232 & 48.3425 & $4.13 \mathrm{E}-01$ & 266 & 55.9722 & $2.97 \mathrm{E}+00$ & 3600 & 53.2782 & 3600 & 106.5564 & $10.2 \%$ & 1 \\
\hline \multicolumn{15}{|c|}{ large-sized instances } \\
\hline 16 & \multirow{3}{*}{80} & 1306 & 407 & 107.0965 & $2.29 \mathrm{E}+00$ & 420 & 118.6993 & $1.86 \mathrm{E}+00$ & 3600 & 112.1208 & 3600 & 188.2985 & $4.7 \%$ & 1 \\
\hline 17 & & 1278 & 433 & 95.9933 & $1.20 \mathrm{E}+00$ & 411 & 102.5468 & $1.84 \mathrm{E}+00$ & 3600 & 198.8833 & 3600 & 137.1635 & $42.9 \%$ & 1 \\
\hline 18 & & 1235 & 472 & 114.1646 & $1.78 \mathrm{E}+00$ & 408 & 123.9676 & $2.72 \mathrm{E}+00$ & 3600 & 112.9421 & 3600 & 147.0629 & $-1.1 \%$ & -1 \\
\hline 19 & \multirow{3}{*}{100} & 1894 & 610 & 904 & 5.36 & 549 & 72.3289 & 2.8 & 3600 & 126.8558 & 3600 & 128.1777 & $92.5 \%$ & 1 \\
\hline 20 & & 2141 & 560 & 157.3409 & 6.74 & 556 & 168.0308 & 7.531 & 3600 & 242.7624 & 3600 & 199.8843 & $27.0 \%$ & 1 \\
\hline 21 & & 2063 & 525 & 120.9239 & $4.75 \mathrm{E}-01$ & 514 & 131.5095 & $3.48 \mathrm{E}+00$ & 3600 & 244.6335 & 3600 & 204.1837 & $68.9 \%$ & 1 \\
\hline 22 & \multirow{3}{*}{150} & 3770 & 937 & 86.9786 & $7.16 \mathrm{E}+00$ & 951 & 151.0684 & $6.39 \mathrm{E}+00$ & 7200 & 285.0914 & 7200 & 154.1776 & $77.3 \%$ & 1 \\
\hline 23 & & 4205 & 943 & 158.4398 & $2.99 \mathrm{E}+00$ & 1175 & 221.2248 & $1.15 \mathrm{E}+01$ & 7200 & 297.4548 & 7200 & 344.0700 & $87.7 \%$ & 1 \\
\hline 24 & & 4590 & 975 & 67.1359 & $3.33 \mathrm{E}+00$ & 1059 & 101.6343 & $5.11 \mathrm{E}+00$ & 7200 & 202.2122 & 7200 & 160.5198 & $139.1 \%$ & 1 \\
\hline 25 & \multirow{3}{*}{200} & \begin{tabular}{|l|}
7704 \\
\end{tabular} & 1448 & 91.0785 & $4.16 \mathrm{E}+00$ & 1456 & 179.9828 & $1.14 \mathrm{E}+01$ & 7200 & $\mathrm{NF}$ & 7200 & 154.1777 & $69.3 \%$ & 1 \\
\hline 26 & & 7546 & 1427 & 165.536 & $1.32 \mathrm{E}+01$ & 1355 & 338.5548 & $1.81 \mathrm{E}+01$ & 7200 & $\mathrm{NF}$ & 7200 & 211.1056 & $27.5 \%$ & 1 \\
\hline 27 & & 7768 & 1502 & 175.6161 & $6.11 \mathrm{E}+00$ & 1489 & 289.4556 & $5.94 \mathrm{E}+00$ & 7200 & $\mathrm{NF}$ & 7200 & 300.0548 & $70.9 \%$ & 1 \\
\hline 28 & & 12027 & 2331 & 102.806 & $4.41 \mathrm{E}+00$ & 2076 & 208.2315 & $9.57 \mathrm{E}+00$ & 7200 & NF & 7200 & 453.7210 & $341.3 \%$ & 1 \\
\hline 29 & 250 & 12134 & 2283 & 149.8248 & $3.72 \mathrm{E}+00$ & 2106 & 227.9566 & $8.40 \mathrm{E}+00$ & 7200 & $\mathrm{NF}$ & 7200 & 195.5056 & $30.5 \%$ & 1 \\
\hline 30 & & 11771 & 1973 & 99.6941 & $9.16 \mathrm{E}+00$ & 2055 & 189.4755 & $1.22 \mathrm{E}+01$ & 7200 & $\mathrm{NF}$ & 7200 & 161.6132 & $62.1 \%$ & 1 \\
\hline 31 & & 30247 & 3030 & 110.9487 & $3.60 \mathrm{E}+00$ & 3049 & 214.449 & $4.23 \mathrm{E}+01$ & 7200 & $\mathrm{NF}$ & 7200 & 234.69037 & $111.5 \%$ & 1 \\
\hline 32 & 400 & 30210 & 2984 & 170.0742 & $7.53 \mathrm{E}+00$ & 3043 & 284.6509 & $7.73 \mathrm{E}+01$ & 7200 & $\mathrm{NF}$ & 7200 & 345.98478 & $103.4 \%$ & 1 \\
\hline 33 & & 29556 & 3020 & 97.5071 & $6.73 \mathrm{E}+00$ & 3105 & 220.0146 & $4.04 \mathrm{E}+01$ & 7200 & $\mathrm{NF}$ & 7200 & 234.14113 & $140.1 \%$ & 1 \\
\hline 34 & & 47029 & 3542 & 121.0351 & $6.15 \mathrm{E}+00$ & 3507 & 208.8335 & $6.22 \mathrm{E}+00$ & 7200 & $\mathrm{NF}$ & 7200 & 215.21219 & $77.8 \%$ & 1 \\
\hline 35 & 500 & 47554 & 3345 & 70.5963 & $4.38 \mathrm{E}+00$ & 3371 & 176.6044 & $3.82 \mathrm{E}+00$ & 7200 & $\mathrm{NF}$ & 7200 & 179.04062 & $153.6 \%$ & 1 \\
\hline 36 & & 48839 & 3437 & 109.0453 & $1.56 \mathrm{E}+00$ & 3498 & 188.5628 & $6.22 \mathrm{E}+00$ & 7200 & $\mathrm{NF}$ & 7200 & 215.21219 & $97.4 \%$ & 1 \\
\hline
\end{tabular}

As shown in Table 5, LINDOGlobal could not find any feasible solutions for problems instances with $n=150,200,250,400$ and 500 (except No. 24). In terms of solution quality, PTbGA is better than or equal to AlphaECP on all large-sized instances except instance No.17. The performance Gap between PTbGA and AlphaECP becomes significantly greater for the instances with $n=400$ and 500. AlphaECP got stuck in local optima for small instances No.1-6, and could not find the optimal solutions. For function $F_{2}$ and when a size of the problem is increased, LINDOGlobal is not able to find any feasible solution even after 7200s. PTbGA has better quality and efficiency than AlphaECP on all large-sized instances except No.16. Also worth noting that, PTbGA is superior than PrGA on all instances using $F_{1}$ and $F_{2}$ functions. 
Table 9: Result comparison of PTbGA with other algorithms using cost function $F_{3 c}$.

\begin{tabular}{|c|c|c|c|c|c|c|c|c|c|c|c|c|c|c|}
\hline \multicolumn{15}{|c|}{ small-sized instances } \\
\hline \multirow{2}{*}{ No. } & \multirow{2}{*}{$n$} & \multirow{2}{*}{$m$} & \multicolumn{3}{|c|}{ PTbGA } & \multicolumn{3}{|c|}{ PrGA } & \multicolumn{2}{|c|}{ LINDOGlobal } & \multicolumn{2}{|c|}{ AlphaECP } & \multirow{2}{*}{ Gap } & \multirow{2}{*}{$h$} \\
\hline & & & $t$ & mean & std & $t$ & mean & std & $t^{\prime}$ & $O B J$ & $t^{\prime}$ & $O B J$ & & \\
\hline 1 & \multirow{3}{*}{5} & 7 & 21 & 17.7868 & $3.65 \mathrm{E}-15$ & 19 & 17.7868 & $3.65 \mathrm{E}-15$ & 13 & 17.7868 & 5 & 17.7868 & $0.0 \%$ & 0 \\
\hline 2 & & 9 & 23 & 17.7868 & $3.65 \mathrm{E}-15$ & 21 & 17.7868 & $3.65 \mathrm{E}-15$ & 104 & 17.7868 & 36 & 17.7868 & $0.0 \%$ & 0 \\
\hline 3 & & 7 & 21 & 17.7868 & $3.65 \mathrm{E}-15$ & 20 & 17.7868 & $3.65 \mathrm{E}-15$ & 14 & 17.7868 & 15 & 17.7868 & $0.0 \%$ & 0 \\
\hline 4 & \multirow{3}{*}{10} & 35 & 45 & 33.6934 & 7.64E-01 & 56 & 35.4376 & $1.46 \mathrm{E}-14$ & 3600 & 32.9299 & 3600 & 45.5650 & $-2.3 \%$ & -1 \\
\hline 5 & & 32 & 70 & 36.3853 & 7.03E-01 & 66 & 42.9655 & $1.46 \mathrm{E}-14$ & 3600 & 35.5299 & 3600 & 44.0590 & $-2.4 \%$ & -1 \\
\hline 6 & & 24 & 59 & 41.3595 & $4.68 \mathrm{E}-01$ & 60 & 52.865 & $0.00 \mathrm{E}+00$ & 3600 & 41.5513 & 3600 & 58.5221 & $0.5 \%$ & 0 \\
\hline \multicolumn{15}{|c|}{ medium-sized instances } \\
\hline 7 & \multirow{3}{*}{20} & 85 & 161 & 55.8904 & $1.60 \mathrm{E}+00$ & 111 & 57.0152 & $0.00 \mathrm{E}+00$ & 3600 & 50.1726 & 3600 & 75.5723 & $-10.2 \%$ & -1 \\
\hline 8 & & 104 & 116 & 44.6081 & $0.00 \mathrm{E}+00$ & 103 & 45.6321 & $1.93 \mathrm{E}+00$ & 3600 & 48.4365 & 3600 & 53.2294 & $8.6 \%$ & 1 \\
\hline 9 & & 93 & 126 & 50.2162 & $0.00 \mathrm{E}+00$ & 115 & 50.2162 & $0.00 \mathrm{E}+00$ & 3600 & 50.2162 & 3600 & 67.5513 & $0.0 \%$ & 0 \\
\hline 10 & \multirow{3}{*}{40} & 263 & 124 & 49.3124 & $1.95 \mathrm{E}+00$ & 151 & 49.4919 & $1.23 \mathrm{E}+00$ & 3600 & 55.2365 & 3600 & 89.5320 & $12.2 \%$ & 1 \\
\hline 11 & & 374 & 174 & 40.4579 & $7.29 \mathrm{E}-15$ & 183 & 46.1681 & $1.15 \mathrm{E}+00$ & 3600 & 47.0284 & 3600 & 75.0300 & $16.2 \%$ & 1 \\
\hline 12 & & 336 & 133 & 55.0127 & $1.31 \mathrm{E}+00$ & 170 & 58.6588 & $3.22 \mathrm{E}+00$ & 3600 & 51.2223 & 3600 & 97.5218 & $-9.6 \%$ & -1 \\
\hline 13 & \multirow{3}{*}{60} & 717 & 214 & 54.9165 & $6.67 \mathrm{E}-01$ & 242 & 56.4198 & $5.61 \mathrm{E}-01$ & 3600 & 54.7797 & 3600 & 241.5177 & $-0.2 \%$ & 0 \\
\hline 14 & & 724 & 255 & 65.2548 & $4.44 \mathrm{E}+00$ & 259 & 67.4152 & $1.88 \mathrm{E}+00$ & 3600 & 67.5076 & 3600 & 138.8497 & $3.5 \%$ & 1 \\
\hline 15 & & 663 & 259 & 37.5143 & $1.55 \mathrm{E}+00$ & 219 & 51.2203 & $1.74 \mathrm{E}+00$ & 3600 & 38.7716 & 3600 & 83.2873 & $3.4 \%$ & 1 \\
\hline \multicolumn{15}{|c|}{ large-sized instances } \\
\hline 16 & \multirow{3}{*}{80} & 1306 & 459 & 70.4999 & $1.95 \mathrm{E}+00$ & 430 & 86.2443 & $1.91 \mathrm{E}+00$ & 3600 & 254.2510 & 3600 & 130.8650 & $85.6 \%$ & 1 \\
\hline 17 & & 1278 & 467 & 75.448 & $3.29 \mathrm{E}+00$ & 463 & 81.3351 & $1.59 \mathrm{E}+00$ & 3600 & 99.9421 & 3600 & 137.2071 & $32.5 \%$ & 1 \\
\hline 18 & & 1235 & 448 & 79.1008 & $4.28 \mathrm{E}+00$ & 470 & 90.531 & $3.70 \mathrm{E}+00$ & 3600 & 106.2355 & 3600 & 144.4630 & $34.3 \%$ & 1 \\
\hline 19 & \multirow{3}{*}{100} & 1894 & 554 & 53.8924 & $9.74 \mathrm{E}-01$ & 577 & 59.1795 & $3.43 \mathrm{E}+00$ & 3600 & 90.2711 & 3600 & 169.4852 & $67.5 \%$ & 1 \\
\hline 20 & & 2141 & 584 & 83.1025 & $0.00 \mathrm{E}+00$ & 628 & 83.9481 & $2.16 \mathrm{E}+00$ & 3600 & 190.7137 & 3600 & 129.8974 & $56.3 \%$ & 1 \\
\hline 21 & & 2063 & 530 & 84.5746 & $1.92 \mathrm{E}+00$ & 544 & 95.6089 & $3.90 \mathrm{E}+00$ & 3600 & 221.1848 & 3600 & 140.2101 & $65.8 \%$ & 1 \\
\hline 22 & \multirow{3}{*}{150} & 3770 & 1142 & 60.9084 & $3.19 \mathrm{E}+00$ & 1091 & 106.6349 & $1.02 \mathrm{E}+01$ & 7200 & 195.9086 & 7200 & 71.9787 & $18.2 \%$ & 1 \\
\hline 23 & & 4205 & 996 & 70.7362 & $2.61 \mathrm{E}+00$ & 1045 & 147.9236 & $6.35 \mathrm{E}+00$ & 7200 & $\mathrm{NF}$ & 7200 & 158.7746 & $124.5 \%$ & 1 \\
\hline 24 & & 4590 & 1043 & 50.1445 & $1.04 \mathrm{E}+00$ & 1168 & 83.7778 & $3.70 \mathrm{E}+00$ & 7200 & $\mathrm{NF}$ & 7200 & 128.9888 & $157.2 \%$ & 1 \\
\hline 25 & \multirow{3}{*}{200} & 7704 & 1503 & 70.6881 & $4.17 \mathrm{E}+00$ & 1609 & 147.6372 & $5.86 \mathrm{E}+00$ & 7200 & $\mathrm{NF}$ & 7200 & 134.6457 & $90.5 \%$ & 1 \\
\hline 26 & & 7546 & 1538 & 99.5808 & $4.39 \mathrm{E}+00$ & 1423 & 260.8419 & $1.44 \mathrm{E}+01$ & 7200 & $\mathrm{NF}$ & 7200 & 336.5219 & $237.9 \%$ & 1 \\
\hline 27 & & 7768 & 1576 & 96.6772 & $4.90 \mathrm{E}+00$ & 1508 & 218.5723 & $8.10 \mathrm{E}+00$ & 7200 & NF & 7200 & 171.0406 & $76.9 \%$ & 1 \\
\hline 28 & & 12027 & 2355 & 90.4287 & $3.96 \mathrm{E}+00$ & 2375 & 185.9695 & $1.53 \mathrm{E}+01$ & 7200 & $\mathrm{NF}$ & 7200 & 132.9645 & $47.0 \%$ & 1 \\
\hline 29 & 250 & 12134 & 2378 & 94.4638 & $4.46 \mathrm{E}+00$ & 2280 & 179.9448 & $1.17 \mathrm{E}+01$ & 7200 & $\mathrm{NF}$ & 7200 & 182.2233 & $92.9 \%$ & 1 \\
\hline 30 & & 11771 & 2058 & 67.5253 & $4.79 \mathrm{E}+00$ & 2208 & 160.9879 & $5.34 \mathrm{E}+00$ & 7200 & $\mathrm{NF}$ & 7200 & 165.0782 & $144.5 \%$ & 1 \\
\hline 31 & & 30247 & 3057 & 62.2175 & $4.12 \mathrm{E}+00$ & 3094 & 144.0554 & $5.24 \mathrm{E}+01$ & 7200 & NF & 7200 & 177.57258 & $185.4 \%$ & 1 \\
\hline 32 & 400 & 30210 & 3080 & 109.1538 & $8.18 \mathrm{E}+00$ & 3175 & 245.5707 & $7.20 \mathrm{E}+01$ & 7200 & $\mathrm{NF}$ & 7200 & 305.65278 & $180.0 \%$ & 1 \\
\hline 33 & & 29556 & 3042 & 55.0122 & $5.12 \mathrm{E}+00$ & 3114 & 137.442 & $5.14 \mathrm{E}+01$ & 7200 & NF & 7200 & 170.58882 & $210.1 \%$ & 1 \\
\hline 34 & & 47029 & 3554 & 77.4947 & $4.84 \mathrm{E}+00$ & 3585 & 164.1868 & $1.00 \mathrm{E}+01$ & 7200 & $\mathrm{NF}$ & 7200 & 171.37157 & $121.1 \%$ & 1 \\
\hline 35 & 500 & 47554 & 3515 & 57.7303 & $5.27 \mathrm{E}+00$ & 3529 & 136.1355 & $1.57 \mathrm{E}+00$ & 7200 & $\mathrm{NF}$ & 7200 & 137.66396 & $138.5 \%$ & 1 \\
\hline 36 & & 48839 & 3495 & 61.6489 & $2.07 \mathrm{E}+00$ & 3537 & 136.921 & $2.94 \mathrm{E}+00$ & 7200 & $\mathrm{NF}$ & 7200 & 138.79592 & $125.1 \%$ & 1 \\
\hline
\end{tabular}

Table 10: Comparing PTbGA's performances with LINDOGlobal and AlphaECP on $F_{1}, F_{2}, F_{3 a}, F_{3 b}, F_{3 c}$.

\begin{tabular}{l|c|c|c|c}
\hline \hline & Small-sized & medium-sized & Large-sized & Total score \\
\hline Functions & W-D-L & W-D-L & W-D-L & W-D-L \\
\hline$F_{1}$ & $0-6-0$ & $0-7-2$ & $14-6-1$ & $14-19-3$ \\
\hline$F_{2}$ & $0-6-0$ & $3-6-0$ & $17-3-1$ & $20-15-1$ \\
\hline$F_{3 a}$ & $0-4-2$ & $2-7-0$ & $16-4-1$ & $18-15-3$ \\
\hline$F_{3 b}$ & $0-6-0$ & $5-0-4$ & $20-0-1$ & $25-6-5$ \\
\hline$F_{3 c}$ & $0-4-2$ & $5-2-2$ & $21-0-0$ & $26-6-4$ \\
\hline
\end{tabular}

\subsection{Robustness}

To examine if PTbGA is robust to the different shapes of a cost function, we use cost function $F_{3}$ (Eq. 7), choosing three different values for the 
parameter $w$ in Eq. 7. As shown in Fig. 11c, by increasing the parameter $w$ from 1 , to 2 and 3, the number of peaks and valleys (local optima) are increased gradually in functions $F_{3 b}, F_{3 c}$. Dealing with these cost functions will be a challenging task. A robust optimisation algorithm should be able to handle this sort of highly non-convex shaped cost functions, without degrading their performances. As can be seen from Tables 7, 8, 9, LINDOGlobal has increasing difficulty in finding feasible solutions on instances with 150, 200, 250, 400 and 500 nodes. Furthermore, on all large-sized instances PTbGA outperforms AlphaECP using functions $F_{3 a}, F_{3 b}$ and $F_{3 c}$. It is evident that the mathematical solvers are sensitive to the non-convex shapes introduced in the cost functions $F_{3 a}, F_{3 b}$, and $F_{3 c}$. In contrast, PTbGA's performance is much more robust with respect to these cost functions.

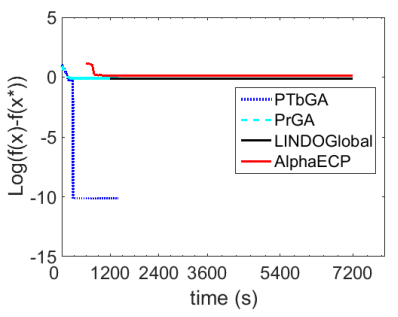

(a) $F_{2}$ on instance No.25.

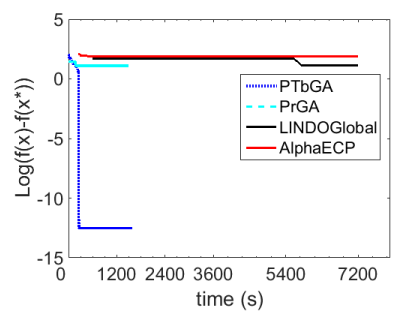

(b) $F_{3 a}$ on instance No.27.

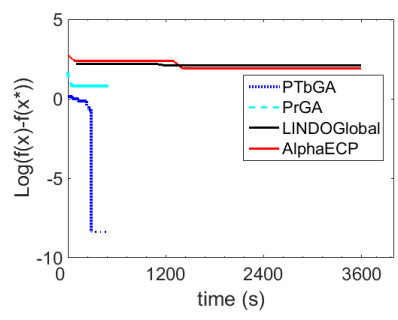

(c) $F_{3 b}$ on instance No.21.

Figure 14: Convergence graphs for PTbGA, PrGA, LINDOGlobal and AlphaECP.

\subsection{Efficiency}

The convergence speeds of PTbGA on large-sized problem instances as compared to that by PrGA and mathematical solvers are presented in Fig. 14. As shown in Fig. 14a, LINDOGlobal cannot find any feasible solution until about 1200s. Once a feasible solution is found by LINDOGlobal, it cannot be further improved and the convergence curve becomes a straight line (Figs. 14a). Similar results are observed for LINDOGlobal considering functions $F_{3 a}$ and $F_{3 b}$ (Figs. 14b,c). Although AlphaECP can find a feasible solution faster than LINDOGlobal, the found solution cannot be improved dramatically within the time limit. Unlike the mathematical approach, PTbGA and PrGA are able to converge faster. In particular, PTbGA is able to find better quality solutions than that of PrGA (Fig. 14). 


\section{Conclusion}

In this paper, we have proposed a probabilistic tree-based representation (PTbR) and incorporated it into a genetic algorithm (PTbGA) for solving large-scale MCFP using nonlinear non-convex cost functions. After identifying the drawbacks of the well-known priority-based representation scheme, we have proposed the PTbR by introducing an improved decoding procedure that allows a more thorough sampling of the search space. This new representation scheme PTbR can be executed $\mathrm{N}$ times (i.e., decoding $\mathrm{N}$ times) to allow for better exploration of the feasible search space of an MCFP. This is in sharp contrast to the limitations imposed by the priority-based representation scheme $(\mathrm{PbR})$. We have conducted systematic and extensive experiments to compare the performance of the PTbR-based GA (PTbGA) variant, the PbR-based GA (PrGA) and two mathematical solver packages for solving MCFP using nonlinear cost functions. In order to identify the optimal parameter settings for both PTbGA and PrGA, the Taguchi method has been applied. PTbGA, PrGA, LINDOGlobal, and AlphaECP have been evaluated on a set of 36 randomly generated MCFP instances considering various nonlinear non-convex cost functions. Our results demonstrate that PTbGA outperforms PrGA on all instances using different cost functions. Unlike mathematical solvers that either got stuck in local optima or cannot find any feasible solutions after 2 hours for large-scale instances, PTbGA can still find high-quality solutions without sacrificing on efficiency. As shown in convergence graphs (Fig. 14), PTbGA converges to a better solution extremely faster than PrGA and mathematical solver packages. Furthermore, PTbGA is more robust to the different shapes of a cost function, as compared to LINDOGlobal and AlphaECP.

\section{References}

[1] R. K. Ahuja, T. L. Magnanti, J. B. Orlin, Network flows: theory, algorithms, and applications, Prentice Hall , pp.4-6, 1993 (1993).

[2] D. B. Fontes, J. F. Gonçalves, Heuristic solutions for general concave minimum cost network flow problems, Networks 50 (1) (2007) 67-76 (2007).

[3] M. S. Monteiro, D. B. Fontes, F. A. Fontes, Concave minimum cost network flow problems solved with a colony of ants, Journal of Heuristics 19 (1) (2013) 1-33 (2013). 
[4] L. A. Vegh, A strongly polynomial algorithm for a class of minimumcost flow problems with separable convex objectives, SIAM Journal on Computing 45 (5) (2016) 1729-1761 (2016).

[5] A. Sifaleras, Minimum cost network flows: Problems, algorithms, and software, Yugoslav Journal of Operations Research 23 (1) (2013) 3-17 (2013).

[6] P. Kovács, Minimum-cost flow algorithms: an experimental evaluation, Optimization Methods and Software 30 (1) (2015) 94-127 (2015).

[7] S. Yan, Y. Shih, C. Wang, An ant colony system-based hybrid algorithm for square root concave cost transhipment problems, Engineering Optimization 42 (11) (2010) 983-1001 (2010).

[8] F. Xie, R. Jia, Nonlinear fixed charge transportation problem by minimum cost flow-based genetic algorithm, Computers \& Industrial Engineering 63 (4) (2012) 763-778 (2012).

[9] A. S. Amiri, S. A. Torabi, R. Ghodsi, An iterative approach for a bilevel competitive supply chain network design problem under foresight competition and variable coverage, Transportation Research Part E: Logistics and Transportation Review 109 (2018) 99-114 (2018).

[10] A. Trivedi, D. Srinivasan, S. Biswas, T. Reindl, A genetic algorithmdifferential evolution based hybrid framework: case study on unit commitment scheduling problem, Information Sciences 354 (2016) 275-300 (2016).

[11] H. R. Bouchekara, A. Chaib, M. A. Abido, R. A. El-Sehiemy, Optimal power flow using an improved colliding bodies optimization algorithm, Applied Soft Computing 42 (2016) 119-131 (2016).

[12] K. Ayan, U. Kllı̧, Artificial bee colony algorithm solution for optimal reactive power flow, Applied soft computing 12 (5) (2012) 1477-1482 (2012).

[13] G. M. Guisewite, P. M. Pardalos, Algorithms for the single-source uncapacitated minimum concave-cost network flow problem, Journal of Global Optimization 1 (3) (1991) 245-265 (1991). 
[14] D. B. Fontes, E. Hadjiconstantinou, N. Christofides, A dynamic programming approach for solving single-source uncapacitated concave minimum cost network flow problems, European Journal of Operational Research 174 (2) (2006) 1205-1219 (2006).

[15] D. B. Fontes, E. Hadji constantinou, N. Christofides, A branch-andbound algorithm for concave network flow problems, Journal of Global Optimization 34 (1) (2006) 127-155 (2006).

[16] R. E. Burkard, H. Dollani, P. T. Thach, Linear approximations in a dynamic programming approach for the uncapacitated single-source minimum concave cost network flow problem in acyclic networks, Journal of Global Optimization 19 (2) (2001) 121-139 (2001).

[17] M. S. Monteiro, D. B. Fontes, F. A. Fontes, An ant colony optimization algorithm to solve the minimum cost network flow problem with concave cost functions, in: Proceedings of the 13th annual conference on Genetic and evolutionary computation, ACM, 2011, pp. 139-146 (2011).

[18] Y.-H. Zhang, Y.-J. Gong, T.-L. Gu, Y. Li, J. Zhang, Flexible genetic algorithm: A simple and generic approach to node placement problems, Applied Soft Computing 52 (2017) 457-470 (2017).

[19] G. Aiello, G. La Scalia, M. Enea, A multi objective genetic algorithm for the facility layout problem based upon slicing structure encoding, Expert Systems with Applications 39 (12) (2012) 10352-10358 (2012).

[20] F. G. Tari, Z. Hashemi, A priority based genetic algorithm for nonlinear transportation costs problems, Computers \& Industrial Engineering 96 (2016) 86-95 (2016).

[21] B. Ghasemishabankareh, M. Ozlen, X. Li, K. Deb, A genetic algorithm with local search for solving single-source single-sink nonlinear non-convex minimum cost flow problems, Soft Computing (2019) 1-17 (2019).

[22] T. Senjyu, K. Shimabukuro, K. Uezato, T. Funabashi, A fast technique for unit commitment problem by extended priority list, IEEE Transactions on Power Systems 18 (2) (2003) 882-888 (2003). 
[23] C. Chung, H. Yu, K. P. Wong, An advanced quantum-inspired evolutionary algorithm for unit commitment, IEEE Transactions on Power Systems 26 (2) (2011) 847-854 (2011).

[24] C. W. Ahn, R. S. Ramakrishna, A genetic algorithm for shortest path routing problem and the sizing of populations, IEEE transactions on evolutionary computation 6 (6) (2002) 566-579 (2002).

[25] N. Shimamoto, A. Hiramatsu, K. Yamasaki, A dynamic routing control based on a genetic algorithm, in: IEEE International conference on neural networks, IEEE, 1993, pp. 1123-1128 (1993).

[26] M. Lotfi, R. Tavakkoli-Moghaddam, A genetic algorithm using prioritybased encoding with new operators for fixed charge transportation problems, Applied Soft Computing 13 (5) (2013) 2711-2726 (2013).

[27] J. J. d. M. Mendes, J. F. Gonçalves, M. G. Resende, A random key based genetic algorithm for the resource constrained project scheduling problem, Computers \& Operations Research 36 (1) (2009) 92-109 (2009).

[28] V. Van Peteghem, M. Vanhoucke, A genetic algorithm for the preemptive and non-preemptive multi-mode resource-constrained project scheduling problem, European Journal of Operational Research 201 (2) (2010) 409-418 (2010).

[29] M. Gen, R. Cheng, L. Lin, Network models and optimization: Multiobjective genetic algorithm approach, Springer Science \& Business Media, 2008 (2008).

[30] D. B. Fontes, J. F. Gonçalves, A multi-population hybrid biased random key genetic algorithm for hop-constrained trees in nonlinear cost flow networks, Optimization Letters 7 (6) (2013) 1303-1324 (2013).

[31] C. Dang, Y. Sun, Y. Wang, Y. Yang, A deterministic annealing algorithm for the minimum concave cost network flow problem, Neural networks 24 (7) (2011) 699-708 (2011).

[32] B. Ghasemishabankareh, M. Ozlen, F. Neumann, X. Li, A probabilistic tree-based representation for non-convex minimum cost flow problems, in: Parallel Problem Solving from Nature-PPSN XV: 15th International 
Conference, Coimbra, Portugal on 8-12 September 2018, Proceedings, Springer, 2018 (2018).

[33] D. E. Goldberg, K. Deb, A comparative analysis of selection schemes used in genetic algorithms, in: Foundations of genetic algorithms, Vol. 1, Elsevier, 1991, pp. 69-93 (1991).

[34] J.-E. Lee, M. Gen, K.-G. Rhee, Network model and optimization of reverse logistics by hybrid genetic algorithm, Computers \& Industrial Engineering 56 (3) (2009) 951-964 (2009).

[35] U. Klanšek, Solving the nonlinear discrete transportation problem by minlp optimization, Transport 29 (1) (2014) 1-11 (2014).

[36] U. Klanšek, M. Pšunder, Solving the nonlinear transportation problem by global optimization, Transport 25 (3) (2010) 314-324 (2010).

[37] Z. Michalewicz, G. A. Vignaux, M. Hobbs, A nonstandard genetic algorithm for the nonlinear transportation problem, ORSA Journal on Computing 3 (4) (1991) 307-316 (1991).

[38] S. Burer, A. N. Letchford, Non-convex mixed-integer nonlinear programming: a survey, Surveys in Operations Research and Management Science 17 (2) (2012) 97-106 (2012).

[39] Y. Lin, L. Schrage, The global solver in the lindo api, Optimization Methods \& Software 24 (4-5) (2009) 657-668 (2009).

[40] T. Westerlund, R. Pörn, Solving pseudo-convex mixed integer optimization problems by cutting plane techniques, Optimization and Engineering 3 (3) (2002) 253-280 (2002).

[41] M. Tawarmalani, N. V. Sahinidis, A polyhedral branch-and-cut approach to global optimization, Mathematical Programming 103 (2005) 225-249 (2005).

[42] R. Al-Aomar, et al., A ga-based parameter design for single machine turning process with high-volume production, Computers \& Industrial Engineering 50 (3) (2006) 317-337 (2006). 
[43] F. Jabbarizadeh, M. Zandieh, D. Talebi, Hybrid flexible flowshops with sequence-dependent setup times and machine availability constraints, Computers \& Industrial Engineering 57 (3) (2009) 949-957 (2009).

[44] B. Ghasemishabankareh, N. Shahsavari-Pour, M.-A. Basiri, X. Li, A hybrid imperialist competitive algorithm for the flexible job shop problem, in: Australasian Conference on Artificial Life and Computational Intelligence, Springer, 2016, pp. 221-233 (2016).

[45] M. Hajiaghaei-Keshteli, S. Molla-Alizadeh-Zavardehi, R. TavakkoliMoghaddam, Addressing a nonlinear fixed-charge transportation problem using a spanning tree-based genetic algorithm, Computers \& Industrial Engineering 59 (2) (2010) 259-271 (2010).

[46] R. Al-Aomar, Incorporating robustness into genetic algorithm search of stochastic simulation outputs, Simulation Modelling Practice and Theory 14 (3) (2006) 201-223 (2006).

[47] M. S. Phadke, Quality engineering using robust design, Prentice Hall PTR, 1995 (1995).

[48] J.-T. Tsai, W.-H. Ho, T.-K. Liu, J.-H. Chou, Improved immune algorithm for global numerical optimization and job-shop scheduling problems, Applied Mathematics and Computation 194 (2) (2007) 406-424 (2007).

[49] B. Naderi, M. Zandieh, A. K. G. Balagh, V. Roshanaei, An improved simulated annealing for hybrid flowshops with sequence-dependent setup and transportation times to minimize total completion time and total tardiness, Expert systems with Applications 36 (6) (2009) 9625-9633 (2009).

[50] S. Bolboacă, L. Jäntschi, Design of experiments: Useful orthogonal arrays for number of experiments from 4 to 16, Entropy 9 (4) (2007) 198-232 (2007).

[51] S. Molla-Alizadeh-Zavardehi, M. Hajiaghaei-Keshteli, R. TavakkoliMoghaddam, Solving a capacitated fixed-charge transportation problem by artificial immune and genetic algorithms with a prüfer number representation, Expert Systems with Applications 38 (8) (2011) 10462-10474 (2011). 